\title{
Identification of herbal categories active in pain disorder subtypes by machine learning help reveal novel molecular mechanisms of algesia
}

DOI:

10.1016/j.phrs.2020.104797

\section{Document Version \\ Accepted author manuscript}

Link to publication record in Manchester Research Explorer

Citation for published version (APA):

Nenadic, G. (2020). Identification of herbal categories active in pain disorder subtypes by machine learning help reveal novel molecular mechanisms of algesia. Pharmacological Research, [104797].

https://doi.org/10.1016/j.phrs.2020.104797

\section{Published in:}

Pharmacological Research

\section{Citing this paper}

Please note that where the full-text provided on Manchester Research Explorer is the Author Accepted Manuscript or Proof version this may differ from the final Published version. If citing, it is advised that you check and use the publisher's definitive version.

\section{General rights}

Copyright and moral rights for the publications made accessible in the Research Explorer are retained by the authors and/or other copyright owners and it is a condition of accessing publications that users recognise and abide by the legal requirements associated with these rights.

\section{Takedown policy}

If you believe that this document breaches copyright please refer to the University of Manchester's Takedown Procedures [http://man.ac.uk/04Y6Bo] or contact uml.scholarlycommunications@manchester.ac.uk providing relevant details, so we can investigate your claim.

\section{OPEN ACCESS}




\section{Journal Pre-proof}

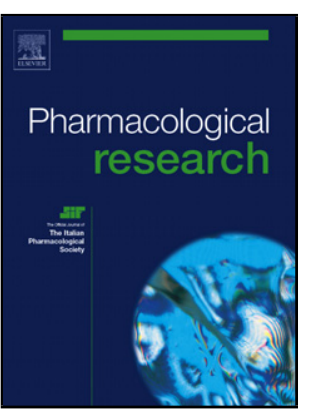

Identification of herbal categories active in pain disorder subtypes by machine learning help reveal novel molecular mechanisms of algesia

Xue Xu, Kuo Yang, FeiLong Zhang, Wenwen Liu, Yinyan Wang, ChangYing Yu, JunYao Wang, Keke Zhang, Chao Zhang, Goran Nenadic, Dacheng Tao, Xuezhong Zhou, Hongcai Shang, Jianxin Chen

PII: S1043-6618(20)31105-1

DOI: https://doi.org/10.1016/j.phrs.2020.104797

Reference: YPHRS 104797

To appear in: Pharmacological Research

Received Date: 16 October 2019

Revised Date: 26 March 2020

Accepted Date: 29 March 2020

Please cite this article as: Xu X, Yang K, Zhang F, Liu W, Wang Y, Yu C, Wang J, Zhang K, Zhang C, Nenadic G, Tao D, Zhou X, Shang H, Chen J, Identification of herbal categories active in pain disorder subtypes by machine learning help reveal novel molecular mechanisms of algesia, Pharmacological Research (2020), doi: https://doi.org/10.1016/j.phrs.2020.104797

This is a PDF file of an article that has undergone enhancements after acceptance, such as the addition of a cover page and metadata, and formatting for readability, but it is not yet the definitive version of record. This version will undergo additional copyediting, typesetting and review before it is published in its final form, but we are providing this version to give early visibility of the article. Please note that, during the production process, errors may be discovered which could affect the content, and all legal disclaimers that apply to the journal pertain.

(C) 2020 Published by Elsevier. 


\section{Identification of herbal categories active in pain disorder subtypes by machine learning help reveal novel molecular mechanisms of algesia}

Xue $\mathrm{Xu}^{1,2 \#}$, Kuo Yang ${ }^{3,4 \#}$, FeiLong Zhang ${ }^{1,5 \#}$, Wenwen $\mathrm{Liu}^{3}$, Yinyan Wang ${ }^{3}$, Chang Ying $\mathrm{Yu}^{5}$, JunYao Wang ${ }^{5}$, Keke Zhang ${ }^{5}$, Chao Zhang ${ }^{6}$, Goran Nenadic ${ }^{7}$, Dacheng Tao ${ }^{8}$, Xuezhong Zhou ${ }^{3 *}$, Hongcai Shang ${ }^{1 *}$, Jianxin Chen ${ }^{5 *}$

${ }^{1}$ Key Laboratory of Chinese Internal Medicine of Ministry of Education and Beijing, Dongzhimen Hospital, Beijing University of Chinese Medicine, Beijing 100700, China

${ }^{2}$ Marcus Institute for Aging Research, Hebrew SeniorLife and Harvard Medical School, Boston, MA 02131, USA

${ }^{3}$ School of Computer and Information Technology, Beijing Jiaotong University, Beijing 100044, China

${ }^{4}$ MOE Key Laboratory of Bioinformatics, TCM-X Centre/Bioinformatics Division, BNRIST/Department of Automation, Tsinghua University, Beijing 10084, China

${ }^{5}$ Beijing University of Chinese Medicine, Beijing 100029, China

${ }^{6}$ School of Mathematical Sciences, Dalian University of Technology, DaLian, Liaoning, 116024, China

${ }^{7}$ Computer Science, Faculty of Engineering and Physical Sciences, University of Manchester, Manchester, UK

${ }^{8}$ School of Information Technologies, The university of Sydney, Darlington, NSW, 2008, Australia

\#These authors are contributing equally to this work.

*Correspondence should be addressed to: xzzhou@bjtu.edu.cn; cjx@bucm.edu.cn; shanghongcai@foxmail.com.

\section{Graphical abstract}
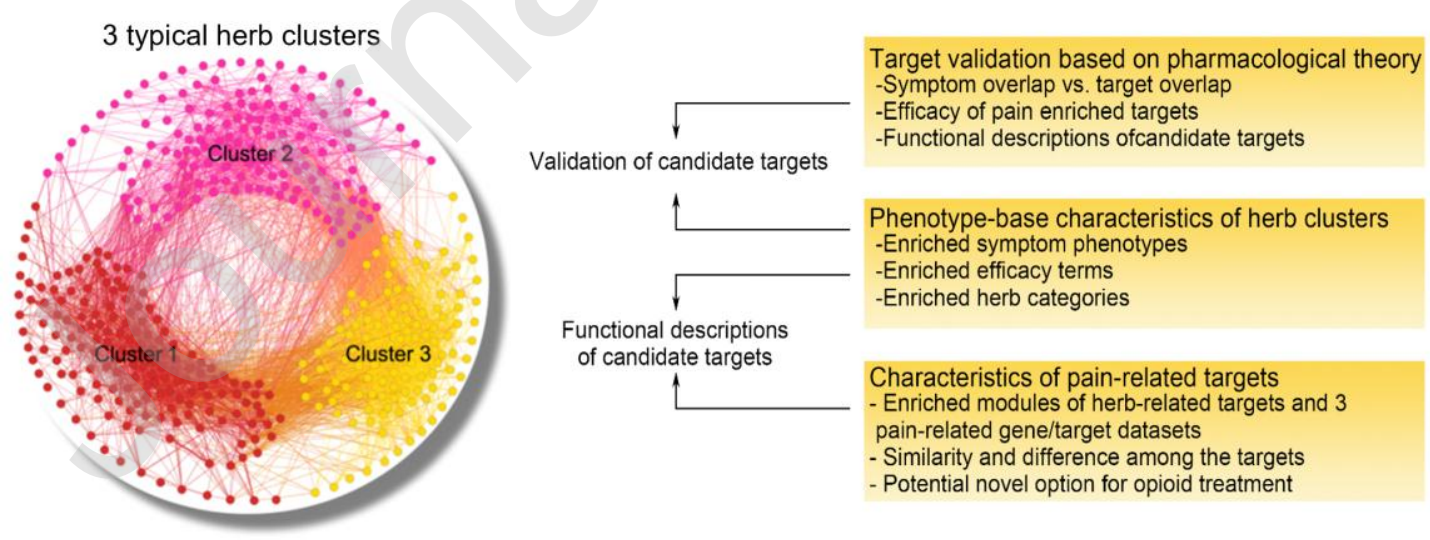

\section{Abstract}

Chronic pain is highly prevalent and poorly controlled, of which the accurate 
underlying mechanisms need be further elucidated. Herbal drugs have been widely used for controlling various pain disorders. The systematic integration of pain herbal data resources might be promising to help investigate the molecular mechanisms of pain phenotypes. Here, we integrated large-scale bibliographic literatures and wellestablished data sources to obtain high-quality pain relevant herbal data (i.e. 426 pain related herbs with their targets). We used machine learn method to identify three distinct herb categories with their specific indications of symptoms, targets and enriched pathways, which were characterized by the efficacy of treatment to the chronic cough related neuropathic pain, the reproduction and autoimmune related pain, and the cancer pain, respectively. We further detected the novel pathophysiological mechanisms of the pain subtypes by network medicine approach to evaluate the interactions between herb targets and the pain disease modules. This work increased the understanding of the underlying molecular mechanisms of pain subtypes that herbal drugs are participating and with the ultimate aim of developing novel personalized drugs for pain disorders.

Keywords: pain disorder subtypes; complex network analysis; network pharmacology; cancer pain

\section{Introduction}

As a major public health concern, pain is a leading contributor to the global burden of many diseases and is one of the most expensive conditions, e.g., a total amount of $\$ 87.6$ billion was spent only for the low back and neck pain in 2013 in USA[1]. Most previous research has focused on opioids, nonsteroidal anti-inflammatory drugs 
(NSAIDs) and marijuana for pain management[2-5]. It is well documented that an array of molecular mechanisms are involved in the development and maintenance of chronic pain, including neuronal plasticity, microglial and astrocyte activation, and immunecell infiltration[6], and represent attractive targets for pharmacological intervention[7, 8]. For example, opioid receptor family responds to classical opioid alkaloids such as morphine and heroin as well as to endogenous peptide ligands like endorphins to regulate pain[9]. NSAIDs (e.g., aspirin and indomethacin) exert their effect of pain relief by inhibiting COX-1 and COX-2 enzymes[10]. However, due to the clinical phenotype heterogeneity of pain disorders, effective therapies for one group patients have a limited or little effect on many other patients[11]. Therefore, elucidating the underlying mechanisms of patient subgroups or phenotype subtypes of pain disorders and identifying the novel drugs without tolerance would be critical for precision medicine.

Traditional Chinese medicine (TCM) is clinical-based medicine with the focus on personalized treatment for individualized patients. Thus individualized patients of the same diseases (e.g., migraine) could obtain a precise diagnosis and optimal treatment from different TCM physicians[12, 13]. The herbal prescriptions used in TCM clinical settings consist of multiple ingredients mainly derived from natural plants. Many chemical drugs were extracted from herbal medicine[14], for example, artemisinin, discovered from Artemisia annua with efficacy and safety for the treatment of malaria[15]. TCM has a long-time clinical practice with herbal medicine (e.g., Yan 
Husuo and Chuan Xiong ) for pain management[16, 17].

The theory and method of network pharmacology $[18,19]$ and machine learning methods [20] are powerful tools to unveil the molecular mechanisms of human diseases [21] and design new drugs[22-24]. With the availability of the large-scale biomedical data (e.g., phenotype-genotype associations and human interactomes), it would deliver promising chances to discover novel drugs for pain treatment using network pharmacology approaches, which would help to develop novel drugs derived from herbal plants with more tailored treatment for pain disorders.

In our study, a unified network pharmacology framework was established to identify herbal categories active in pain disorder subtypes, which would help reveal novel molecular mechanisms of algesia (Figure 1). First, we identified the literature-based associations between the active molecules and the therapeutical effects from TCM herbs by literature mining method. Then, the binding targets of these active herbal molecules with human and gut microorganism targets were then explored by the interaction simulations. Second, three pain related herb categories from 2040 herb pharmacopoeia records were obtained by using network clustering algorithm (section 4.1) based on the constructed herb-herb network. Further measurement of interactions among the distinct targets of the three herb categories to the disease modules of pain phenotypes has been taken, and thereby the underlying molecular mechanisms of the three herb categories for pain regulations was explored. Moreover, the close relationship between the targets of herbs and pain was revealed by the network analysis 
A

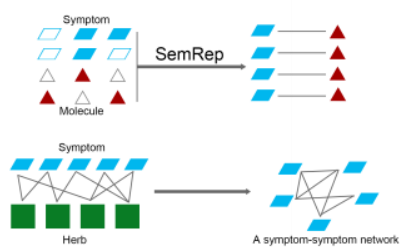

D

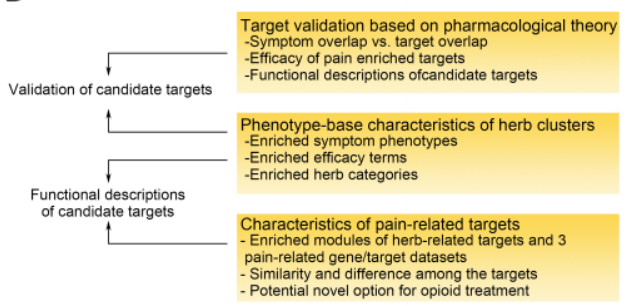

B

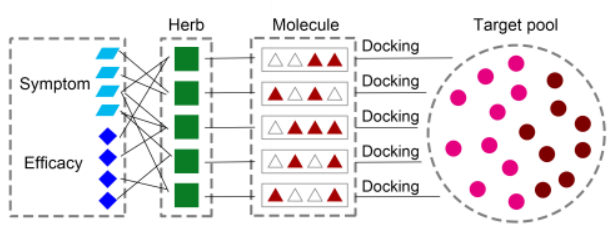

C

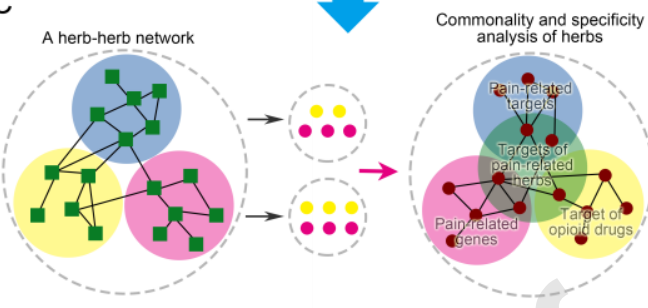

Figure 1. A flow chart for identification of herbal categories active in pain disorder subtypes. (A) Co-occurrence between herbal molecules and phenotypes from SemRep and the construction of a symptom-symptom network based on herb-symptom associations. (B) Construction of the small molecule-related targets by docking analysis. (C) Construction of an herb-herb network based on herb-symptom/efficacy associations and the identification of 3 herb clusters and 3 respective target clusters. (D) Mechanism analysis of the pain-related herbs and targets.

of the targets of herbs and pain-related genes. Our findings suggest that integrated network pharmacology analysis of both TCM and modern biomedical data has the utility to novel drug discovery with precise treatment for pain disorders.

\section{Results}

\subsection{Published active herbal molecules related to herb-related phenotypes}

To curate the high reliable active molecules derived from herbal medicines, we manually collected 965 phenotypes (Data S1, Table S1) from the indication sections of 2040 herb medicines in Pharmacopoeia of the People's Republic of China (PPRC) (Section 4.1), and extracted 1978 herbal molecules (Data S2) from TCM-related database (supplemental material (SM) section 1.1-1.2). After that, we obtained 6,892 phenotype-herb molecule co-occurrences (Data S3) from SemRep[25] (Section 4.2, SM section 1.3), yielding a total of 24,616 literatures, and 593 phenotypes and 406 herbal molecules (Figure 2A). The results showed that the phenotypes that has co-occurrences 
with herbal molecules are very concentrated. The most frequent 5 phenotypes (Figure 2B) are neoplasm, inflammation, wounds and injuries, obesity, and seizures, which have hundreds of co-occurrence literatures. The quality of the retrieved relationships was evaluated by TCM experts (SM section 1.4, Data S4). Finally, 406 herbal molecules as active molecules with therapeutic effects were obtained. 
A

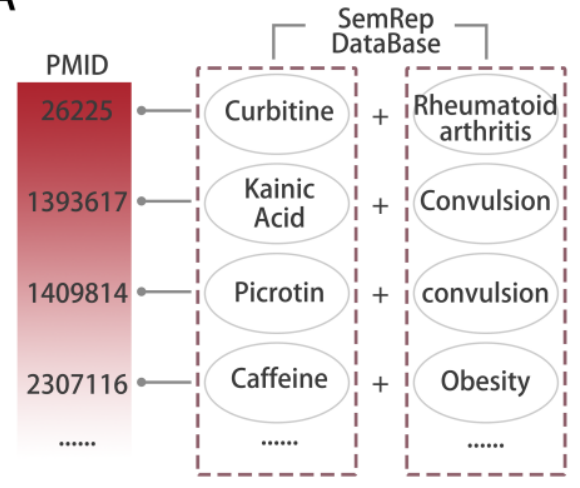

C

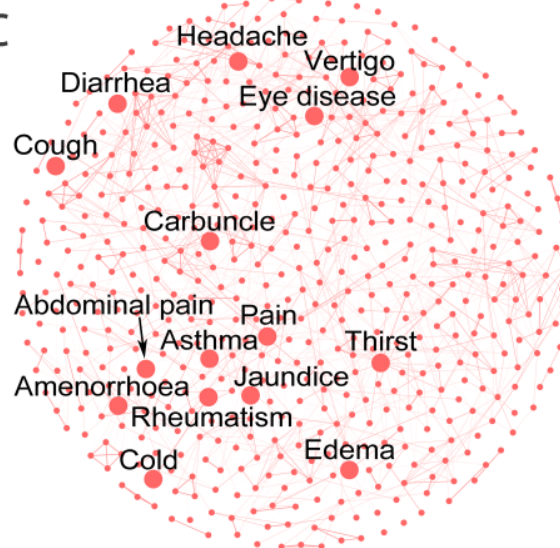

B
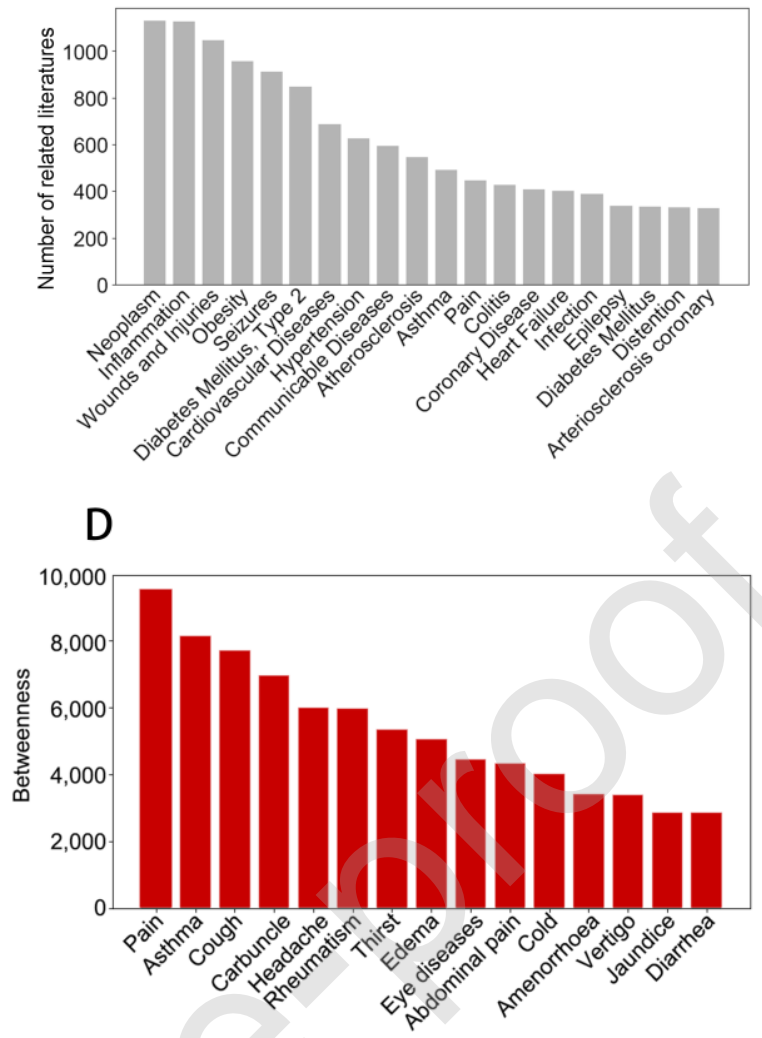

$E$

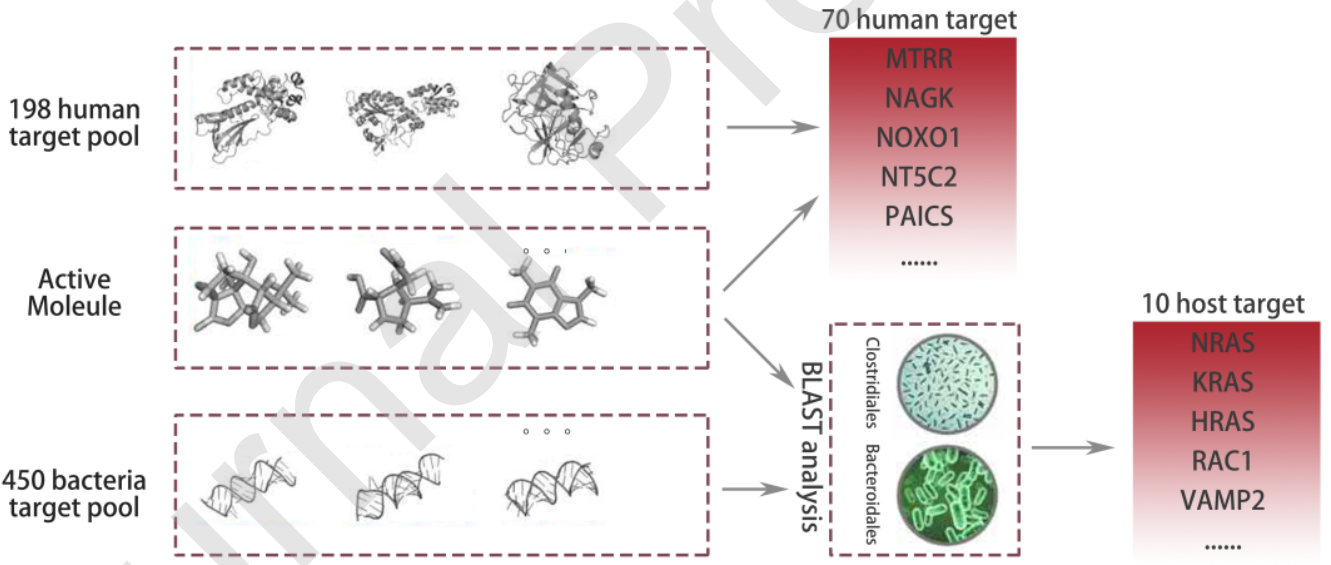

Figure 2. Identification of active herbal molecules and targets. (A) 406 active herbal molecules retrieved from co-occurrence between herbal molecules and phenotypes by in SemRep database; (B) Identification of 20 most frequent phenotypes from the co-occurrences; (C) A phenotype-phenotype network constructed based on 474 herbs; (D) Top 15 phenotypes with highest betweenness derived from the phenotype-phenotype network; (E) Docking analysis for the interactions between active molecules and bacteria 16S rRNA/human proteins. BLAST analysis supports identification of Clostridiales and Bacteroidales from the screened RNA sequences, and the corresponding host targets are collected from PATRIC database and literature.

To further investigate the indications of the 474 herbs containing the 406 herbal molecules, we extracted the phenotypes represented in the indications of herb records 
in PPRC. We constructed a phenotype-phenotype network, which contained 563 phenotypes and 3,038 edges (the weights of edges represent the degree of shared herbs between phenotypes and the edges are filtered by its weight $\geqq 0.8$, Figure $2 \mathrm{C}$ and $2 \mathrm{D}$ ). We found that the 15 phenotypes with highest betweenness, are mostly pain related phenotypes, such as pain, asthma, cough, carbuncle, headache and rheumatism. This indicated that the main efficacies of the 474 herbs are relevant to the treatment for pain phenotypes. And these phenotypes are related to more herbs than other phenotypes in PPRC. For example, the top 5 phenotypes (Figure S1) with most related herbs are pain, carbuncle, cough, asthma and edema, which also have high node's degree and betweenness in the phenotype-phenotype network.

\subsection{Novel drug targets of the active herbal molecules}

To further identify the novel drug targets of the active molecules, we collected 198 human proteins with their 3D structures (named as the Human Target Pool, HTP) from DrugBank[26] (SM section 2.1). The 406 active herbal molecules were docked to each protein in HTP (Section 4.3, Figure 2E), and a list of 70 proteins are obtained by strict filtering (Section 4.3, Data S5). Next, 3D structures of 450 16S rRNA (named as Bacteria Target Pool, BTP) were extracted from Human Microbiome Project (HMP) (Section4.3, SM 2.4) [27, 28]. Finally, the corresponding 10 host proteins were associated the active herbal molecules with by docking and BLAST analysis (Section

\section{3, SM section 2.4, Data S6).}

As a tacit assumption in pharmacology, drugs with similar drug efficacies and 
A

\begin{tabular}{|c|c|c|c|}
\hline \multirow{3}{*}{$\begin{array}{c}\text { sympton-gene } \\
\text { relationship }\end{array}$} & \multirow{3}{*}{$\begin{array}{l}\text { relationships } \\
\text { between herb } \\
\text { and symptom } \\
\text { from PPRC }\end{array}$} & $\begin{array}{l}\text { relationships between herbal } \\
\text { molecules and human and host target }\end{array}$ & \multirow{3}{*}{$\begin{array}{c}\text { relationships } \\
\text { between herbs and } \\
\text { herbal molecule from } \\
\text { TCM-related database }\end{array}$} \\
\hline & & 1 & \\
\hline & & $\begin{array}{c}\text { relationships between herbal } \\
\text { molecule and extend target based on PPI }\end{array}$ & \\
\hline & & $\downarrow$ & 1 \\
\hline \multicolumn{2}{|c|}{$\begin{array}{l}\text { herb-gene relationships after } \\
\text { chi-square test }\end{array}$} & \multicolumn{2}{|c|}{ herb-target relationships } \\
\hline & & $\downarrow$ & \\
\hline
\end{tabular}

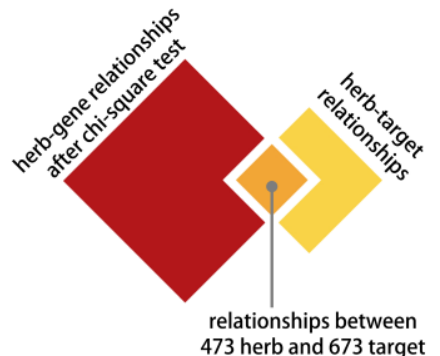

B

\begin{tabular}{c|c|}
$\begin{array}{c}\text { Belidonium } \\
\text { Majus }\end{array}$ & $\begin{array}{c}\text { Rhododendron } \\
\text { simsii Planch }\end{array}$ \\
\hline $\begin{array}{c}\text { Cough } \\
\text { Asthma }\end{array}$ & $\begin{array}{c}\text { Cough } \\
\text { Asthma } \\
\text { Breathe Heavliy }\end{array}$ \\
$\begin{array}{c}\text { Breathe Heavily } \\
\text { Contracture and } \\
\text { Pain of Gastric } \\
\text { Cacity }\end{array}$ & $\begin{array}{c}\text { Excedssive } \\
\text { Sputum }\end{array}$ \\
&
\end{tabular}

\begin{tabular}{c|cc} 
Chelidonium & Plantago Asiatica \\
Majus & Cough Ologuresis \\
\hline Cough & Asthma \\
$\begin{array}{c}\text { Breathe Heavily } \\
\text { Contracture and } \\
\text { Pain of Dastric } \\
\text { Cavity }\end{array}$ & $\begin{array}{c}\text { Diarrhea } \\
\text { Carbuncle Edema } \\
\text { Difficulty and Pain } \\
\text { Spitting blood } \\
\text { Summer-heat and Damp }\end{array}$ \\
\hline
\end{tabular}
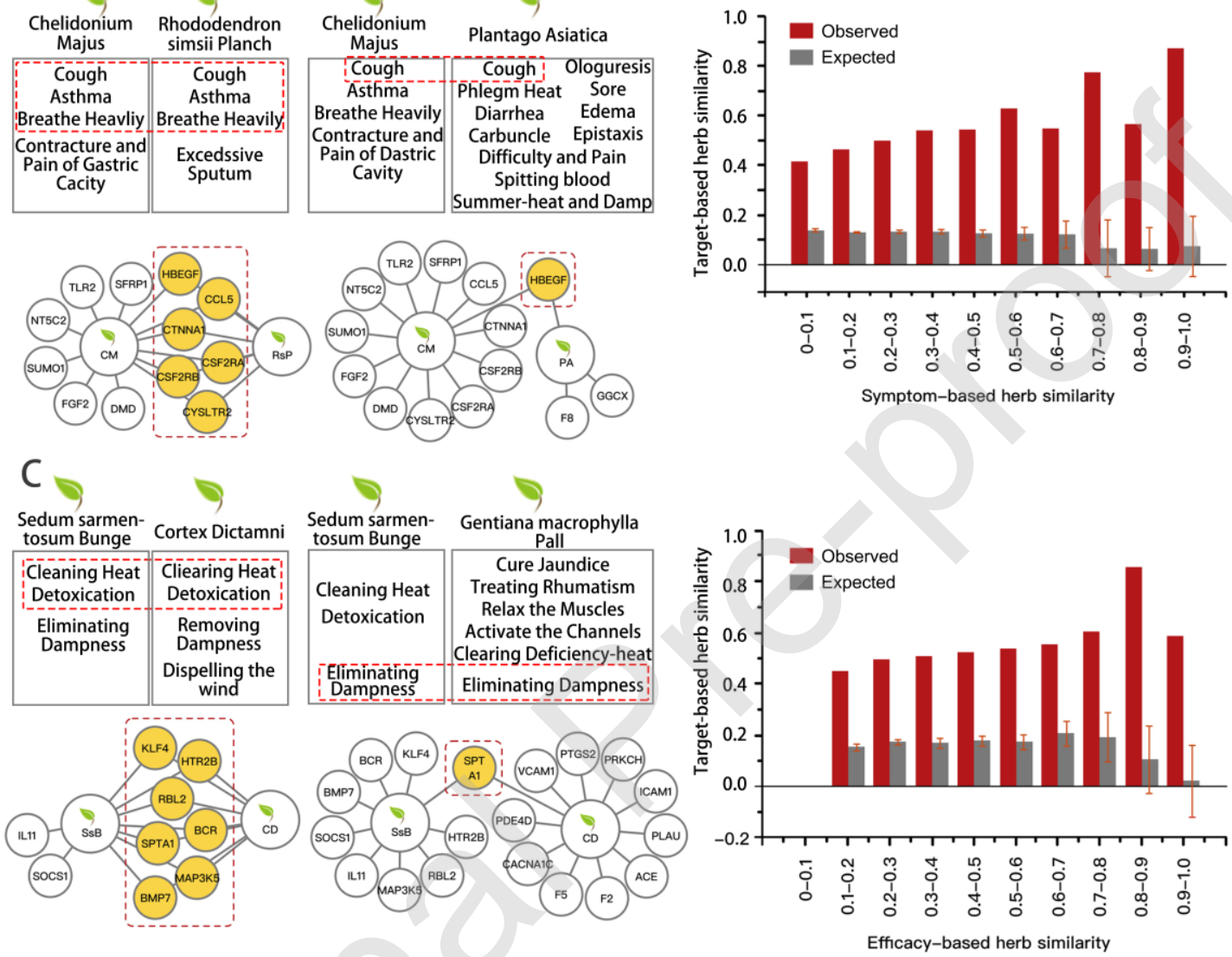

Figure 3. Target validation by tacit pharmacological assumption "drugs with similar drug efficacy and phenotypes share similarly biological targets". (A) Construction of relationships between herbs and targets for calculation of target similarities; (B) Drugs with similar drug efficacy share similar targets. The similarity of herb efficacy for each pair of herbs was calculated and divided into 10 intervals. For the herb pairs involved in each subinterval, the similarity of their corresponding targets is measured. Red and black lines show the mean values of target similarity of herbs and random controls, respectively; (C) Drugs with similar symptoms share similar targets. The similarity of symptoms for each pair of herbs was calculated and divided into 10 intervals. For the herb pairs involved in each subinterval, the similarity of their corresponding targets is measured. Orange and blue bars show the mean values of target similarity of herbs and random controls, respectively.

indicated phenotypes were considered to share similar biological targets[29]. To validate the reliability of the obtained drug targets (SM section 3.1, Figure 3A), we conducted the method described in Zhou et al.[21] to construct two herb networks, namely symptom-based herb network (SHN) and target-based herb network (THN), in 
which the links representing shared symptoms and shared targets (Section 4.4), respectively. The overlapping link ratio of two networks shows positive correlation with herb similarity $(\mathrm{PCC}=0.16$, p-value $<2.2 \mathrm{E}-16$; Figure $3 \mathrm{~B})$, that is, herbs with more similar treated symptoms are more likely to have common target associations (Tables S5 and S6). Similarly, we found that herbs with more similar efficacies are more likely to have common target associations ( $\mathrm{PCC}=0.11$, p-value $<3.6 \mathrm{E}-4$; Figure $3 \mathrm{C}$, Table $\mathrm{S} 7$ ). For example, the two herbs Chelidonium Majus (Bai Qucai) and Rhododendron simsii Planch (Ying Shanhong) that have shared treated phenotypes (e.g. cough and asthma) have the shared targets (e.g. HBEGF and CCF5), as well as the two herbs Chelidonium Majus (Ying Shanhong) and Plantago Asiatica (Che Qianzi). These two validations proved the reliability of the novel herb-target associations.

Further analysis showed out of the 1636 small drug-related targets in DrugBank, only $224(13.7 \%)$ were related to pain relief (Figure 4(A)), while among the 70 human targets, $45(64.3 \%)$ were associated with pain phenotypes (Figure 4(B)). Also, the remaining proteins in the HTP were found to be highly pain-related (Figure 4(C)). The results indicated that the proteins in the HTP were mainly pain-related.

In addition, pathway enrichment analysis (SM section 3.2) showed the human and host targets were mainly enriched in (Table S9): (1) pain-related pathways, e.g., arachidonic acid metabolism and purine metabolism; (2) immune-related pathways, e.g., B cell receptor signaling pathway and natural killer cell mediated cytotoxicity. Further pathways comparison of the human and host targets and the 128 remaining proteins 

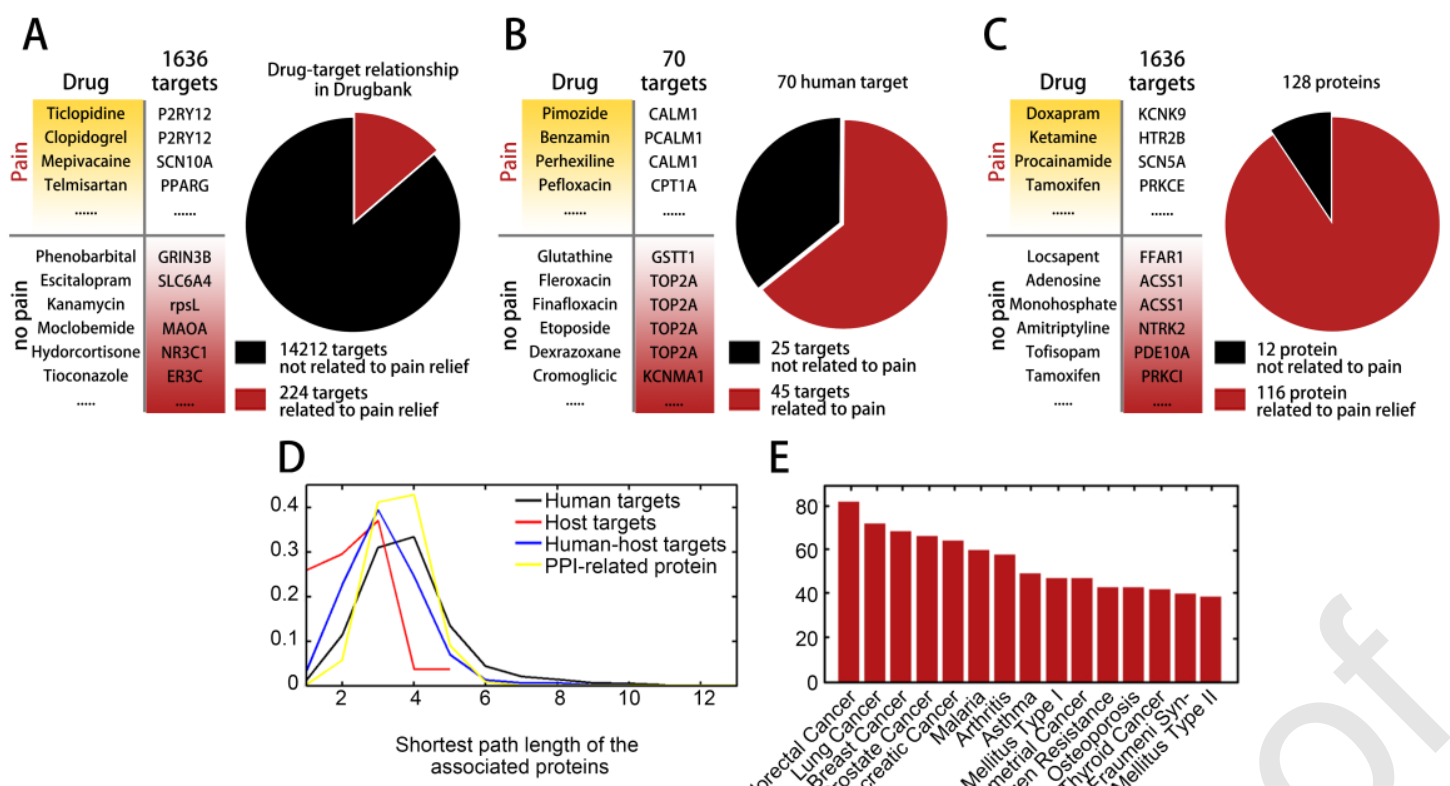

E

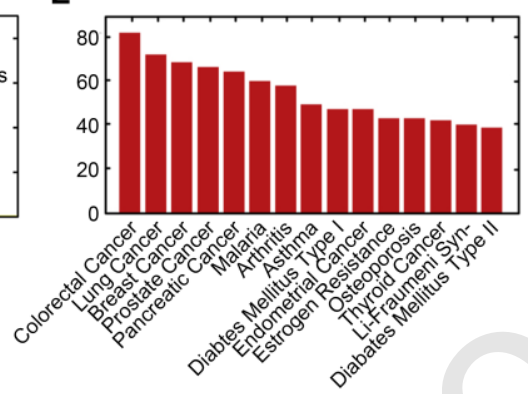

Figure 4. Characteristics of candidate targets. (A) 224 pain related genes derived from 6696 relationships between small drugs and targets in DrugBank; (B) 45 pain related targets out of the 70 human targets; (C) 116 pain related proteins out of the 128 remaining proteins in the human target pool; (D) Shortest path analysis for the 70 human targets and the 10 host targets; (E) 15 intestinal bacteria-related diseases with the highest frequency derived from the corresponding diseases of 474 herbs from MalaCards.

(Table S10) in the HTP implied that the herbal targets with various functions besides

the pain-related functions could be screened out if a larger 3D protein database was

available. Comparison results of the average shortest path length (ASPL) between the human and the host targets in the PPI network (Section 4.7) showed the close relationships among targets (Figure 4D).

To investigate the properties of 10 host targets, we extended the relationships between herbal molecules and host targets based on STRING PPIs, and collected their interactions with diseases from MalaCards[30]. The highest-frequent diseases were shown in Figure 4E, indicating that the herbs had the potential to treat immune-related diseases via the impacts on Clostridiales and Bacteroidales.

\subsection{Pain-related herb network and herb categories}


Systematic reviews showed that less than one-third of patients with pain obtained sufficient pain relief with current pharmacological treatments[31, 32], such poor response probably reflect failure to target the individually relevant pain-generating mechanisms, which are obviously different within the same clinical entity.

To explore the different herb-related pain phenotypes, we screened 426 pain related herbs from the 474 herbs based on the indication sections of herbs in PPRC. To assess the treatment scope of the pain related herbs, 573 corresponding phenotypes were collected from the SemRep database and were compared with those of the PPRC herb medicines (Figure S1). The result showed that the 2 lists of phenotypes achieved good consistency, especially the high frequent phenotypes, which suggested that the pharmacological effects of pain related herbs in PPRC were actively investigated.

To identify the distinct efficacies and symptoms of the 426 herbs, an herb-herb network, where nodes $(n=426)$ represented herbs, and links $(n=25,126)$ represented the shared symptoms or efficacies, was constructed. The herbs with higher centrality (i.e., degree and betweenness) would have more similar herbs than those with lower centrality. For example, for the hub node: Angelicae Sinensisis (AS, Dang Gui, betweenness=618), its first neighbors involved Carthami Flos (Hong Hua) used in TCM to promote blood circulation and to alleviate pain, Myrrha (Mo Yao) mainly for the treatment of chest pain, epigastric pain and dysmenorrhea, and Ligusticum chuanxiong Hort. (Chuan Xiong) for the treatment of headaches, abdominal pain, and menstrual disorder, etc. Interestingly, the herb pairs with similar treated phenotypes or efficacies 
would be commonly prescribed for similar disease conditions. For example, the pair of

A. sinensis and C. Flos was commonly used to promote blood circulation, remove blood stasis and relieve pain by TCM practitioners[33], the pair of A. sinensis and Myrrha was used to activate blood and relieve swelling and pain, and the pair of A. sinensis and L. chuanxiong was used to nourish blood and relieve rheumatism and cold[31].

To further identify the distinct herb clusters with similar treated symptoms or efficacies, we adopted an efficient community detection method to find the herb categories used for treatment of pain phenotypes (section 4.6, SM section 4.1). Finally, we detected 3 herb communities/clusters (i.e. herb categories, HCs) from the whole herb network, for which there are 146 herbs for $\mathrm{HC} 1,165$ herbs for HC2, and 115 herbs for HC3 (Figure 5A, Data S7). For example, HC2 contains Radix Paeoniae Rubra (Chi Shao, betweenness=0.77), Myrrha (betweenness=0.76), Strychnos Nuxvomica $(\mathrm{Ma}$ Qianzi, betweenness=0.71), Aristolochia Fangchi (Fang Ji, betweenness $=0.60)$ as the main herbs with top high centralities.

\subsection{Significant efficacies and molecular characteristics associated to herb} categories

To identify the phenotypic features (i.e. PPRC indications and efficacies) involved in each herb category, the number of corresponding herbs in the category and in the pain related herbs were collected respectively, and the difference between them was evaluated by binomial test and risk ratio (Section 4.2). The enrichment results (Table 1) indicated that the phenotypes in $\mathrm{HCl}$ were mainly related to the indications cough and 
(B) Analysis of herb clusters

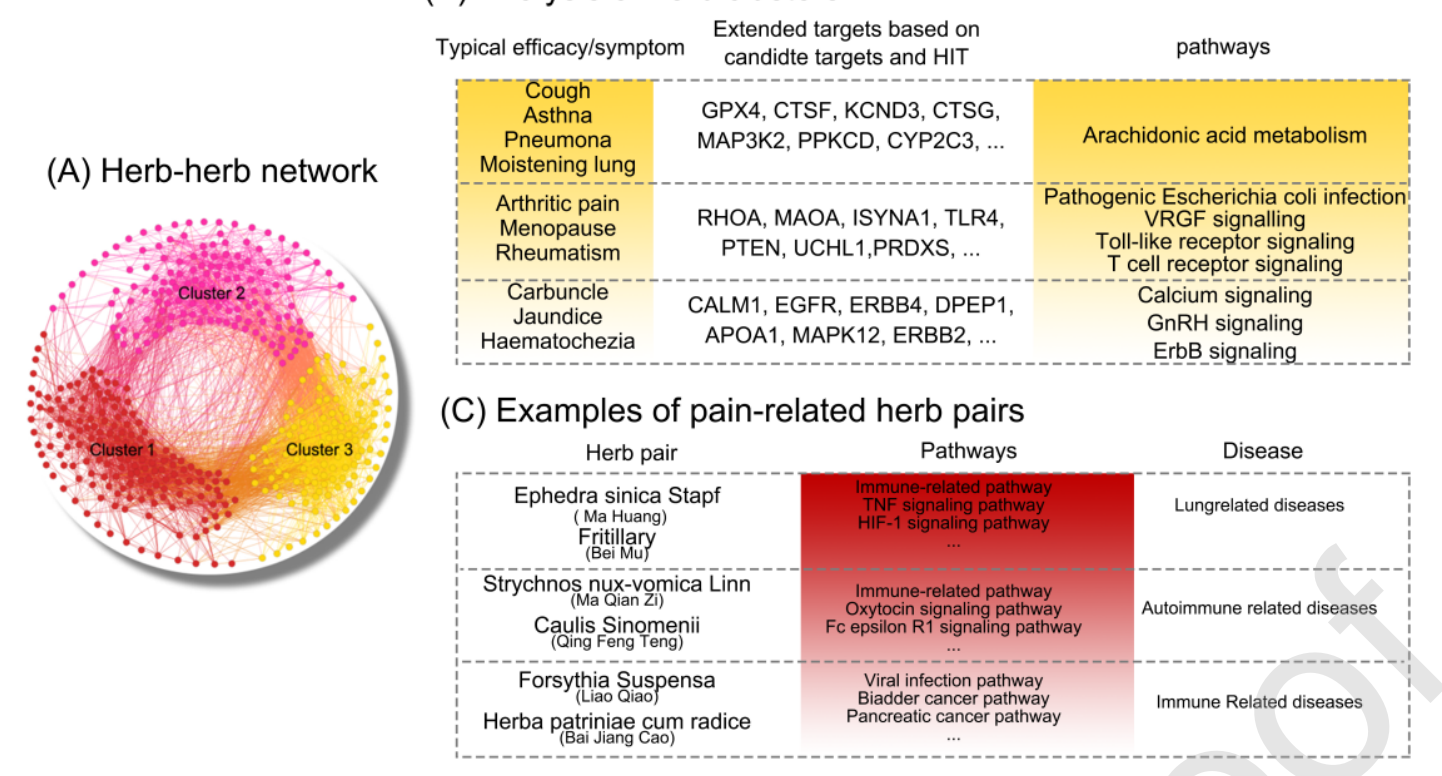

Figure 5. Phenotypes of pain-related herbs. (A) Construction of a herb-based network based on symptom/efficacy similarities of pain-related herbs to identify 3 clusters of pain-related herbs with typical symptoms/efficacy; (B) Analysis of the extended targets of the 3 types of herbs; (C) Representative herb examples derived the herb-based network to validate the different phenotypes of pain.

asthma, and related to the efficacy relieving cough; the phenotypes in HC2 were closely associated with the indications arthritic pain, menopause, rheumatism, and related to the efficacy eliminating the wind-damp; the phenotypes in HC3 were closely related to the indications carbuncle, jaundice, haematochezia, and related to the efficacy clearing heat. The phenotype distinctions of the 3 HCs validated the reliability of herb clustering methods (Figure 5B).

We further investigated the enriched targets of each herb category (Section 4.8). Because of incompleteness for docked targets $(n=80)$ of the herbs, we integrated docked targets and targets from HIT, which lead to 27,393 herb-target relationships including 426 distinct herbs and 1027 targets. Finally, 12, 10 and 20 typical targets were obtained for $\mathrm{HC} 1, \mathrm{HC} 2$ and $\mathrm{HC} 3$, respectively (SM section 4.3, Data S8). Based on the typical targets of each HCs, we also obtained the enriched pathways of these herb categories 
Table 1. Symptom/efficacy enrichment of herb categories (HCs).

\begin{tabular}{|c|c|c|c|c|c|}
\hline \multicolumn{6}{|l|}{ HC1 } \\
\hline Symptom & P-value & Relative risk & Efficacy & P-value & Relative risk \\
\hline Cough & $1.09 \mathrm{E}-12$ & 2.8617 & $\begin{array}{l}\text { Promoting the } \\
\text { growth of body fluid }\end{array}$ & 0.0011 & 2.7094 \\
\hline Asthma & $1.57 \mathrm{E}-11$ & 2.7495 & Relieving a cough & 0.0030 & 2.6747 \\
\hline Pneumonia & $1.70 \mathrm{E}-06$ & 2.8097 & Moistening lung & 0.0041 & 2.9178 \\
\hline Sputum & $7.92 \mathrm{E}-06$ & 2.7962 & - & - & - \\
\hline Thirst & 0.00001 & 2.4176 & - & - & - \\
\hline Constipation & $4.15 \mathrm{E}-05$ & 2.6641 & - & - & - \\
\hline Diabetes mellitus & $5.92 \mathrm{E}-05$ & 2.7719 & - & - & - \\
\hline Thirst & 0.0004 & 2.9178 & - & - & - \\
\hline Productive cough & 0.0009 & 2.9178 & - & - & - \\
\hline \multicolumn{6}{|l|}{ HC2 } \\
\hline Symptom & P-value & Relative risk & Efficacy & P-value & Relative risk \\
\hline Arthritic pain & 4.29E-08 & 2.4645 & Relieving pain & $3.42 \mathrm{E}-09$ & 2.2806 \\
\hline Menopause & $1.06 \mathrm{E}-07$ & 2.4589 & Activating qi & 0.0002 & 2.36667 \\
\hline Rheumatism & $5.38 \mathrm{E}-07$ & 2.5081 & $\begin{array}{l}\text { Eliminating the } \\
\text { wind-damp }\end{array}$ & 0.0008 & 2.5818 \\
\hline Dysmenorrhea & $1.68 \mathrm{E}-05$ & 2.4862 & Promoting blood & 0.0009 & 1.9024 \\
\hline Abdominal pain & 0.0001 & 2.0522 & Dispelling the wind & 0.0013 & 1.9988 \\
\hline Neoplasm & 0.0005 & 2.4459 & Dredging the channel & 0.0033 & 2.3974 \\
\hline Pain & 0.0017 & 2.1328 & Dredging the channel & 0.0050 & 2.3832 \\
\hline $\begin{array}{l}\text { Menstruation } \\
\text { Disturbances }\end{array}$ & 0.0022 & 2.4097 & 2 & & - \\
\hline Chest pain & 0.0025 & 2.1124 & 28 & - & - \\
\hline Bloated feeling & 0.0039 & 1.9364 & - & - & - \\
\hline \multicolumn{6}{|l|}{ HC3 } \\
\hline Symptom & P-value & Relative risk & Efficacy & P-value & Relative risk \\
\hline Carbuncle & $1.05 \mathrm{E}-05$ & 2.1829 & Clearing heat & $8.76 \mathrm{E}-15$ & 2.5367 \\
\hline Jaundice & $1.86 \mathrm{E}-05$ & 2.7783 & Removing toxic & $1.04 \mathrm{E}-10$ & 2.4696 \\
\hline Haematochezia & 0.0011 & 2.6754 & Stopping bleeding & $5.00 \mathrm{E}-06$ & 2.6031 \\
\hline Snake Bites & 0.0016 & 3.3339 & Cooling blood & 0.0003 & 2.3573 \\
\hline Swelling & 0.0022 & 2.6148 & Detumescence & 0.0011 & 1.9757 \\
\hline Eczema & 0.0054 & 2.3396 & - & - & - \\
\hline Ocular discomfort & 0.0059 & 2.9635 & - & - & - \\
\hline Diarrhea & 0.0076 & 2.4696 & - & - & - \\
\hline Pain & 0.0110 & 2.0938 & - & - & - \\
\hline erysipelas & 0.0114 & 2.8812 & - & - & - \\
\hline
\end{tabular}

(Table 2).

For HC1, PRKCD was critical for the regulation of the chemosensitivity of cancer cells[34]. ADCYAP1 has both proliferative and anti-proliferative effects on cancer cell growth[35]. CTSF and MAP3K2 influenced cancer risk[36, 37], and CTSG contributed to bone resorption and niche preparation for cancer-initiating cells[38]. Pathway 
Table 2 Pathway enrichment of enriched targets for herb categories (HCs).

\begin{tabular}{|c|c|c|c|}
\hline \multicolumn{4}{|l|}{ HC1 } \\
\hline Typical targets (12) & Pathway & P-value & Genes \\
\hline 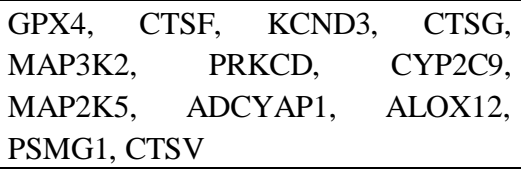 & $\begin{array}{l}\text { Arachidonic } \\
\text { metabolism }\end{array}$ & 0.0024 & CYP2C9, GPX4, ALOX12 \\
\hline \multicolumn{4}{|l|}{ HC2 } \\
\hline Typical targets (21) & Pathway & P-value & Genes \\
\hline \multirow{5}{*}{$\begin{array}{l}\text { RHOA, MAOA, ISYNA1, TLR4, } \\
\text { PTEN, UCHL1, PRDX5, FUS, TIAM2, } \\
\text { MAOB, RB1, HSPB1, CAV1, } \\
\text { NFATC3, TRPV1, EP300, MAPK13, } \\
\text { CD14, NFE2L2, ALB, TEP1 }\end{array}$} & $\begin{array}{l}\text { Pathogenic Escherichia coli } \\
\text { infection }\end{array}$ & 0.0118 & RHOA, TLR4, CD14 \\
\hline & VEGF signaling pathway & 0.0199 & MAPK13, HSPB1, NFATC3 \\
\hline & Prostate cancer & 0.0274 & EP300, RB1, PTEN \\
\hline & $\begin{array}{l}\text { Toll-like receptor signaling } \\
\text { pathway }\end{array}$ & 0.0347 & MAPK13, TLR4, CD14 \\
\hline & $\begin{array}{l}\mathrm{T} \text { cell receptor signaling } \\
\text { pathway }\end{array}$ & 0.0392 & MAPK13, RHOA, NFATC3 \\
\hline \multicolumn{4}{|l|}{ HC3 } \\
\hline Typical targets (11) & Pathway & P-value & Genes \\
\hline \multirow{3}{*}{$\begin{array}{l}\text { CACNA1S, CALM1, MAPK12, } \\
\text { ERBB4, EGFR, DPEP1, APOA1, } \\
\text { ERBB2, DDC, GPLD1, DCT }\end{array}$} & Calcium signaling pathway & 0.0001 & $\begin{array}{l}\text { ERBB4, ERBB2, CALM1, } \\
\text { EGFR, CACNA1S }\end{array}$ \\
\hline & GnRH signaling pathway & 0.0005 & $\begin{array}{l}\text { MAPK12, CACNA1S, } \\
\text { CALM1, EGFR }\end{array}$ \\
\hline & ErbB signaling pathway & 0.0096 & EGFR, ERBB4, ERBB2 \\
\hline
\end{tabular}

analysis further showed that GPX4, CYP2C9 and ALOX12 were enriched in arachidonic acid metabolism. The metabolites of arachidonic acid could promote the pain associated with inflammation and were important mediators in lung cancer process[39]. A number of studies have also reported a positive correlation between the amount of arachidonic acid in inflammatory cells and the risk of bronchial asthma[40].

The result suggested that the efficacy of herbs in $\mathrm{HCl}$ was mainly in the treatment of lung related pain.

The typical targets in $\mathrm{HC} 2$ were enriched in pathogenic Escherichia coli infection, VEGF signaling pathway, prostate cancer, toll-like receptor signaling pathway and $\mathrm{T}$ cell receptor signaling pathway. Infections caused by bacterial pathogens were found to be commonly associated with pain[41]. The direct effect of VEGF on follicular cell 
proliferation and apoptosis[42], and the high expression of VEGF in rheumatoid arthritis[43] were reported. Toll-like receptors were found to be strongly expressed on the surface epithelium of ovaries[44], and their excessive activity induced autoimmune diseases and related acute pain[45]. In addition, T cells had been implicated in pain, especially neuropathic pain[46]. The results indicated that the herbs in HC2 enabled to treat reproduction and autoimmune related pain.

For HC3, the typical targets were enriched in calcium signaling pathway, GnRH signaling pathway and ErbB signaling pathway. Calcium signaling played important roles in carcinogenesis such as transformation of normal cells to cancerous cells, tumor formation and growth, invasion, angiogenesis and metastasis[47]. GnRH signaling directly regulated prostate, breast, endometrial, and ovarian cancer cell proliferation[48]. ErbB receptors, in particular ErbB1 and ErbB2, were important in the development and malignancy of cancers, such as breast and ovarian cancers[49]. Thus, the typical targets in $\mathrm{HC} 3$ were identified to be associated to cancer, which indicated that the efficacy of herbs in $\mathrm{HC} 3$ were mainly to treat immune related cancer pain.

\subsection{Network pharmacological mechanisms of herbs for pain subtypes}

The underlying network mechanisms of disease subtypes could be investigated by the disease module approach[50]. To illustrate the network mechanisms of pain phenotype subtypes, we identified 314 topological modules of human PPI network (Section 4.6) and integrated a well-known pain-gene database with 554 associations and pain drug related 267 targets (Section 4.1, SM section 4.4). 
We obtained 18 significant correlated topological modules to pain genes (PGM) and 15 pain targets enriched modules (PTM, Section 4.8). If the detected herb categories could pharmacologically treat pain related subtypes, we would expect high degree of disease modules to be covered by the targets of these herb categories. Here, we obtained 1,028 targets of the 426 pain related herbs and 30 herb targets enriched modules (HTM, SM 4.4), 37\% (11 modules) of which covered $61 \%(=11 / 18, p$-value $=1.86 \mathrm{e}-08)$ of the 18 PGMs. Moreover, 73\% (=11/15, $p$-value=9.58e-10) PTMs were overlapped with HTM, in which there were 3 disease modules (i.e. M145, M146 and M64) overlapped with PGM as well. In addition, the ASPL between herb-related targets (Htargets) with pain-related genes (Pgenes) and targets (Ptargets) are much smaller than random control, which implied that herb-related targets are closely connected to the pain-related genes/targets in the PPI network. These results indicated the potential of herb targets for pain subtype treatment and the possibility of novel drug development derived from herb ingredients (see Table 3 for the enriched modules and Table S15 for the modules covered by different herb categories).

For example, M145 is a pain disease module ( $p$-value $=7.88 \mathrm{E}-18)$ both covered by herb-related targets ( $p$-value $=7.70 \mathrm{E}-09$, involving targets from all the 3 herb categories) and pain-related targets ( $p$-value $=1.02 \mathrm{E}-14)$, which is also a typical module intervened by opioids drugs ( $p$-value=6.84E-08) (Figure 6D). It showed that there were 19 painrelated genes targeted by herbs in the M145 module, in which CCR7 was the target of Tripterygium wilfordii (Lei Gongteng) (Figure 6B), OPRK1 and CCR5 were the targets 
Table 3 Significant topological PPI modules correlated to the targets of pain-related herbs and the painrelated gene/target datasets. The bold figure shows the $p$ values with significant difference.

\begin{tabular}{|c|c|c|c|c|c|c|c|}
\hline \multicolumn{4}{|l|}{ Module } & \multicolumn{4}{|c|}{ Number of enriched genes; $p$-value } \\
\hline $\begin{array}{l}\text { Module } \\
\text { ID }\end{array}$ & $\begin{array}{l}\text { Number } \\
\text { of nodes }\end{array}$ & $\begin{array}{l}\text { Number } \\
\text { of edges }\end{array}$ & $\begin{array}{l}\text { Network } \\
\text { density }\end{array}$ & Htargets & Pgenes & Ptargets & Otargets \\
\hline M94 & 192 & 765 & 0.042 & $\begin{array}{l}54 ; 5.15 \mathrm{E}- \\
17\end{array}$ & $\begin{array}{l}31 ; 1.12 \mathrm{E}- \\
10\end{array}$ & $11 ; 0.0015$ & - \\
\hline M48 & 191 & 2658 & 0.146 & $\begin{array}{l}52 ; 4.42 \mathrm{E}- \\
16\end{array}$ & $11 ; 0.0731$ & $12 ; 0.0004$ & - \\
\hline M145 & 142 & 6567 & 0.656 & $\begin{array}{l}\text { 33; 7.70E- } \\
09\end{array}$ & $\begin{array}{l}35 ; 7.88 \mathrm{E}- \\
18 \\
\end{array}$ & $\begin{array}{l}23 \text {; 1.02E- } \\
14\end{array}$ & $6 ; 6.84 \mathrm{E}-08$ \\
\hline M103 & 114 & 295 & 0.046 & $\begin{array}{l}26 ; 7.07 E- \\
07\end{array}$ & $6 ; 0.1476$ & - & - \\
\hline M98 & 162 & 635 & 0.049 & $\begin{array}{l}\text { 31; 1.08E- } \\
06\end{array}$ & $14 ; 0.0042$ & - & \\
\hline M97 & 133 & 555 & 0.063 & $\begin{array}{l}27 ; 4.64 \mathrm{E}- \\
06\end{array}$ & $\begin{array}{l}17 ; 4.59 \mathrm{E}- \\
05\end{array}$ & $3 ; 0.2259$ & - \\
\hline M100 & 150 & 889 & 0.08 & $\begin{array}{l}27 ; 6.42 \mathrm{E}- \\
05\end{array}$ & $10 ; 0.0613$ & - & - \\
\hline M143 & 164 & 6931 & 0.519 & $\begin{array}{l}27 ; 8.61 E- \\
05\end{array}$ & $\begin{array}{l}48 ; 6.97 \mathrm{E}- \\
28\end{array}$ & $\begin{array}{l}20 ; 9.39 E- \\
11\end{array}$ & - \\
\hline M194 & 23 & 33 & 0.13 & $8 ; 0.0002$ & $3 ; 0.0551$ & $2 ; 0.0674$ & - \\
\hline M124 & 73 & 1416 & 0.539 & $15 ; 0.0003$ & - & - & - \\
\hline M118 & 55 & 280 & 0.189 & $12 ; 0.0005$ & - & $-\quad$ & - \\
\hline M246 & 48 & 117 & 0.104 & 11; 0.0007 & $6 ; 0.0108$ & $1 ; 0.3721$ & - \\
\hline M126 & 89 & 772 & 0.197 & 16; 0.0011 & $9 ; 0.0091$ & $6 ; 0.0079$ & - \\
\hline M213 & 102 & 1142 & 0.222 & $16 ; 0.0028$ & - & $5 ; 0.0371$ & - \\
\hline M187 & 42 & 107 & 0.124 & 9; 0.0028 & $7 ; 0.0012$ & $8 ; 1.34 \mathrm{E}-06$ & $1 ; 0.0618$ \\
\hline M89 & 17 & 29 & 0.213 & $5 ; 0.0056$ & $1 ; 0.3544$ & $1 ; 0.2412$ & - \\
\hline M125 & 61 & 322 & 0.176 & $10 ; 0.0110$ & - & $3 ; 0.0894$ & - \\
\hline M47 & 200 & 1176 & 0.059 & $23 ; 0.0130$ & $9 ; 0.1302$ & $4 ; 0.1988$ & - \\
\hline M54 & 128 & 526 & 0.065 & $16 ; 0.0167$ & - & - & - \\
\hline M243 & 127 & 691 & 0.086 & $16 ; 0.0176$ & - & - & - \\
\hline M196 & 49 & 76 & 0.065 & $8 ; 0.0196$ & $5 ; 0.0346$ & $1 ; 0.3721$ & - \\
\hline M146 & 154 & 2458 & 0.209 & $18 ; 0.0217$ & $\begin{array}{l}33 ; 3.37 E- \\
15\end{array}$ & $\begin{array}{l}\text { 14; 2.13E- } \\
06\end{array}$ & $2 ; 0.0225$ \\
\hline M197 & 9 & 14 & 0.389 & $3 ; 0.0218$ & $1 ; 0.2602$ & - & - \\
\hline M64 & 184 & 1306 & 0.078 & $20 ; 0.0234$ & $\begin{array}{l}24 ; 2.53 E- \\
07\end{array}$ & $\begin{array}{l}18 ; 1.55 E- \\
08\end{array}$ & 7; 6.71E-09 \\
\hline M193 & 60 & 172 & 0.097 & 9; 0.0235 & $3 ; 0.2185$ & $6 ; 0.0011$ & - \\
\hline M113 & 53 & 209 & 0.152 & 8; 0.0257 & - & - & - \\
\hline M211 & 37 & 183 & 0.275 & $6 ; 0.0359$ & - & - & - \\
\hline M43 & 110 & 625 & 0.104 & $13 ; 0.0394$ & - & - & - \\
\hline M202 & 58 & 213 & 0.129 & $8 ; 0.0404$ & - & 6; 0.0008 & $2 ; 0.0035$ \\
\hline M244 & 94 & 260 & 0.059 & $11 ; 0.0478$ & $6 ; 0.0969$ & - & - \\
\hline
\end{tabular}

of capsule of Papaver somniferum (Ying Suke, the fruit of one of the plants from which opium is derived), and NPY was the target of Tetradium ruticarpum (Wu Zhuyu), all of 

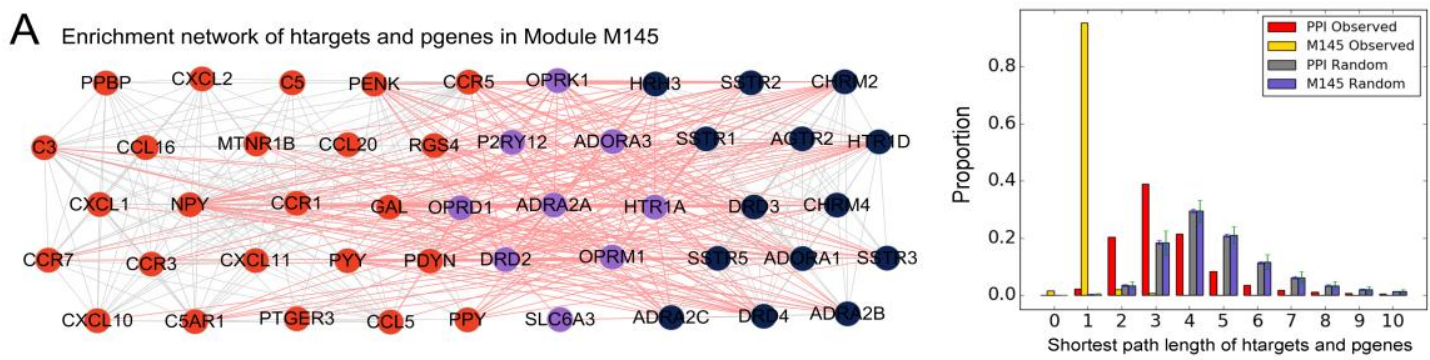

B Enrichment network of htargets and ptargets in Module M145
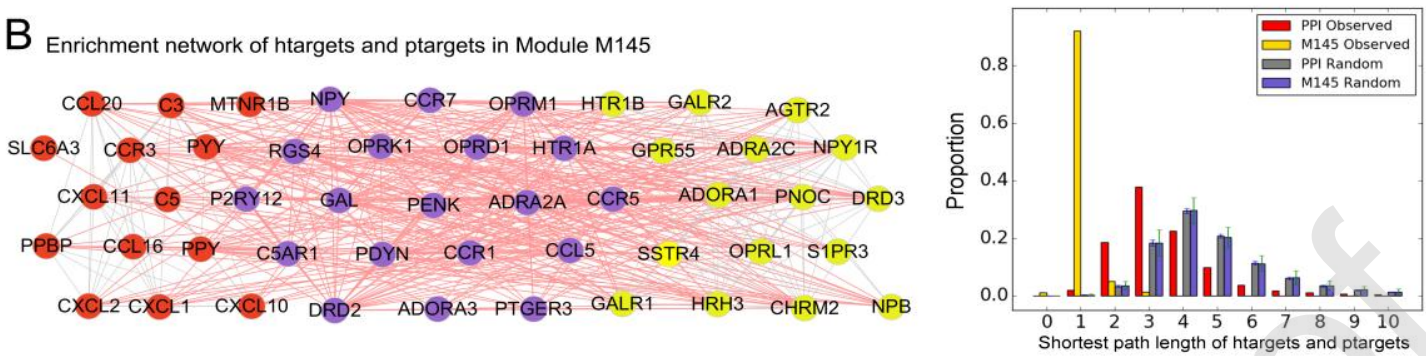

C Enrichment network of htargets and otargets in Module M145
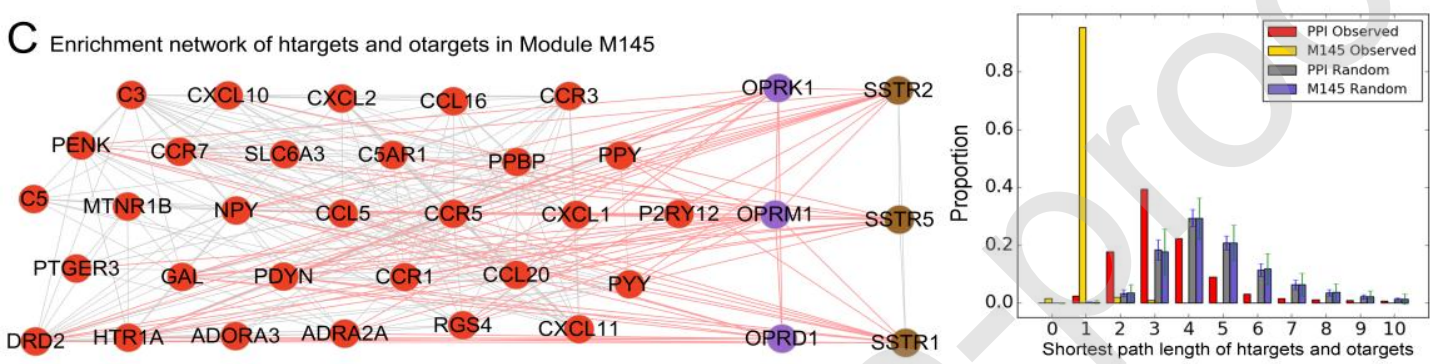

D

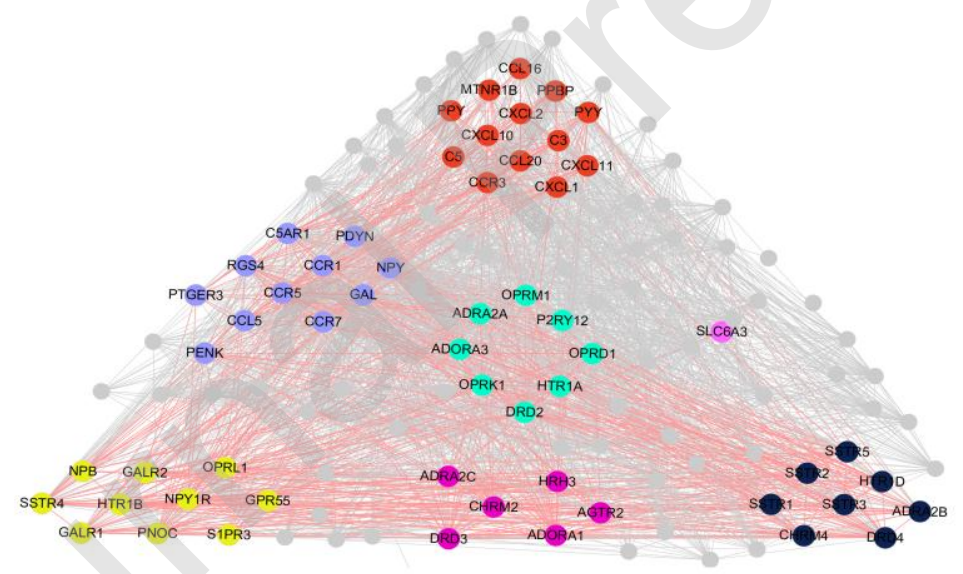

Figure 6. Herb-related targets and pain-related targets/genes in M145 module derived from human PPI network. (A) An amplified enriched network of herb-related targets (Htargets) with pain-related genes (Pgenes). The bar chart on the right side presents the proportion of shortest path length between Htargets and Pgenes compared with random genes; (B) An amplified enriched network of Htargets with pain-related targets (Ptargets). The bar chart on the right side presents the proportion of shortest path length between Htargets and Ptargets compared with random genes; (C) An amplified enriched network of herb-related targets (Htargets) with targets of opioid drugs (Otargets). The bar chart on the right side presents the proportion of shortest path length between Htargets and Otargets compared with random genes; (D) A M145-based network of Htargets, Pgenes, Ptargets and Otargets.

which were representative herbs for pain treatment. In addition, 9 shared targets

between Htargets and Pgenes were found in the M145 module (Figure 6A). For example, 
the HTR1A was the target of Hyoscyamus niger (Tian Xianzi), whose major efficacy was the treatment of stomach/intestinal pain [51], and OPRK1 and OPRM1 were the targets of capsule of P. somniferum . Interestingly, the corresponding western drugs involved alprenolol (a well-known drug used to prevent angina) with HTR1A as the target, morphine, tramadol and codeine (3 powerful opioid drugs) with OPRK1 as the target, and levomethadyl acetate and sufentanil (2 synthetic opioid analgesic drugs) with OPRM1 as the target. The OPRM1 with CHRM2 and CHRM4 were also the targets of hydromorphone (the first line opioid for cancer pain).

Opioid treatment bears the risk of addiction and life-threatening side effects, and patients receiving opioids quickly desensitize and thus require ever-increasing doses[52]. Therefore, therapeutic efficacy of opioid treatment requires urgent improvement[53]. The overlap and interaction between Htargets and Otargets indicated

Table 4 Pathway enrichment analysis of the targets of pain-related herbs (HtargetsHtargets), the pain-related genes/targets (Pgenes/Ptargets) and the targets of opioid drugs (Otargets).

\begin{tabular}{|l|l|l|l|}
\hline Pathways & $\begin{array}{l}\text { Number of } \\
\text { genes }\end{array}$ & P-value & Number of Genes \\
\hline Neuroactive ligand-receptor interaction & 45 & $1.26 \mathrm{E}-40$ & $\begin{array}{l}\text { Htargets: 10; Pgenes: 20; Ptargets: 20; } \\
\text { Otargets: 6 }\end{array}$ \\
\hline Chemokine signaling pathway & 25 & $7.59 \mathrm{E}-19$ & Htargets: 11; Pgenes: 4 \\
\hline Cytokine-cytokine receptor interaction & 22 & $4.69 \mathrm{E}-13$ & Htargets: 9; Pgenes: 4 \\
\hline cAMP signaling pathway & 14 & $4.69 \mathrm{E}-06$ & $\begin{array}{l}\text { Htargets: 4; Pgenes: 8; Ptargets: 8; } \\
\text { Otargets: 3 }\end{array}$ \\
\hline Sphingolipid signaling pathway & 9 & $4.76 \mathrm{E}-04$ & $\begin{array}{l}\text { Htargets: 2; Pgenes: 4; Ptargets: 3; } \\
\text { Otargets: 1 }\end{array}$ \\
\hline Staphylococcus aureus infection & 5 & 0.0192 & Htargets: 3; Pgenes: 1 \\
\hline Regulation of lipolysis in adipocytes & 5 & 0.0361 & Htargets: 2; Pgenes: 4; Otargets: 1 \\
\hline
\end{tabular}

that it was promising to treat pain without resistance by herbs. In fact, the 6 Otargets located in M145 module were closely related to 33 Htargets (with 3 shared targets, 
Figure 6C). GO analysis further showed the Htargets enriched 3 additional pain-related pathways compared with the Otargets (Table 4 and Table S15), indicating the potential of pain-related herbs to overcome opioid resistance.

\section{Discussion}

Pain seriously interferes with the daily life of patients, and the focus on pain can be traced back to $\sim 2000 \mathrm{BC}$. After identifying pain as a symptom of disease, patients have turned to opioids to relieve their pain for thousands of years[54]. However, opioids are well known for their dark side, i.e., craving, dependency, and addiction. From 1999 to 2015, more than 183,000 people in the U.S. died of an opioid painkiller overdose[55]. The question is that the opioid drugs are the only painkillers that work for many people, and thus restricting the use of opioids is almost impossible. Therefore, scientists are focusing on understanding of pain mechanisms to inhibit opioid addition while retaining the drugs' pain-killing power, with the ultimate aim to develop an effective, nonaddictive painkiller.

In this work, we selected PPRC, an official and authoritative compendium of drugs of China to construct a phenotype pool. With the phenotype pool, we retrieved the active herbal molecules from the SemRep database, and identified their corresponding human and host targets based on docking analysis. Further construction of pain-related herb network indicated 3 herb clusters with specific targets and phenotypic features. Moreover, human PPI-based network analysis showed the correlations between the targets of pain-related herbs and well-known pain-related genes/targets. We suggested 
that the completely new strategies would facilitate us to develop herb-based pain-killing drugs without dependency and addiction.

\subsection{Novel indications of herb categories for pain relief}

Because of the complexity of physiology underlying pain, the current therapeutic approach for pain is still a stepwise process to identify which drugs or drug combinations provide the optimized pain relief with fewest side-effects[56, 57]. Drug design for pain regulation from TCM has opened up great opportunities for individualized treatment strategies in relieving pain[58]. The herbs or herb combinations derived from our herb clusters would propose highly biological insights for the underlying mechanisms of more specific pain subtypes and novel pain drug development.

For example, in the $\mathrm{HC1}$, we found there were many herbs used for treating chronic cough, such as Ephedra Sinica Stapf (Ma Huang, betweenness=330.76), which was a herb derived from E. Sinica Stapf and other Ephedra species, commonly used to treat asthma and chronic cough. We obtained 8 active herbal molecules from E. Sinica Stapf, and the 2 active constituents (i.e. ephedrannin A and B) effectively exerted antiinflammatory actions by suppressing the translocation of nuclear factor-kappa B (NF$\kappa \mathrm{B})$ and the phosphorylation of p38 mitogen-activated protein[59] and kinase[60]. The 2 regulators were implicated in many aspects of the airway pathology in asthma[61, 62]. Meanwhile, another herb: Fritillary (Bei Mu, betweenness=6.33, Figure 5C) has been claimed to have remarkable curative effects in clearing inflammations and relieving 
cough. The 14 identified active herbal molecules such as alkaloids pingbeimine C, pingbeininoside, pingbeidinoside and yibeinoside A were reported to relieve cough and asthma[63]. Both of E. Sinica Stapf and fritillary were typical herbs used in various clinical prescriptions to treat chronic cough and bronchial pneumonia. In addition, we found that the main enriched pathways of these 2 herbs involved immune-related pathways, TNF signaling pathway, HIF-1 signaling pathway, etc. TNF contributed to airway inflammation in chronic asthma[61]. HIF-1 was a contributing factor to the pathogenesis of hypoxic pulmonary hypertension[64]. The results showed the treatment of ESS and fritillary chronic cough and chronic lung diseases. It is interesting that the interaction between chronic cough and neuropathic pain has been investigated intensively in recent work, which indicates that chronic cough is likely a neuropathic pain disorder[65] and the regular pain drugs, such as amitriptyline and gabapentin, could be effectively used for chronic cough. Our work indicated that many clinical used herbal medicines for chronic cough might propose novel indications for neuropathic pain subtypes, which have shared molecular mechanisms of particular kind of chronic cough.

In the HC2, the dried seeds of Strychnos nuxvomica (Ma Qianzi, betweenness $=338.36$ ) have been claimed to improve blood circulation and relieve rheumatic pain with 5 screened active herbal molecules. For example, as the major active components of S. nuxvomica, the indole alkaloids brucine and brucine $\mathrm{N}$-oxide showed significant pain modulation and anti-inflammation effects[66]. Another herb 
named Caulis Sinomenii (Qing Feng Teng, betweenness=74.83), which was the dried plant stems of Sinomenium acutum and Sinomenium acutum var. cinereum, has been widely used for relieving symptom of rheumatosis, blood segregation and pain in TCM clinical settings. As one of the active molecules of C. Sinomenii, the alkaloid sinomenine showed antinociceptive effects to alleviate the symptoms of rheumatic diseases[67]. Both of S. nuxvomica and C. Sinomenii were widely prescribed in clinical formulae to treat rheumatism in China. The enriched pathways of these 2 herbs involved natural killer cell mediated cytotoxicity pathway, oxytocin signaling pathway, Fc epsilon RI signaling pathway, etc., most of which would devote to the underlying mechanisms of rheumatoid arthritis[68-70].

For the HC3, Forsythia suspense (Lian Qiao, betweenness=378.364) was derived a flowering plant, which was used to treat fever, carbuncle and disperse lumps[71]. As the main bioactive components of F. suspense, phillyrin and forsythiaside had vasorelaxant[72] and anti-inflammatory activities[73]. Forsythoside B exhibited significant cytotoxic activity against cancer cells[74]. Another herb with similar efficacies in HC3 was Herba Patriniae (Bai Jiang Cao, betweenness=44.66, Figure 5C), which was commonly used for heating-clearing, detoxicating, and pain relief. Both of F. suspense and H. Patriniae were heat-clearing medicinal, which were regularly used in clinical formulas to treat carbuncle and swollen poison. Their enriched pathways contained viral infection pathways, bladder cancer pathway, pancreatic cancer pathway, prostate cancer pathway, proteoglycans in cancer pathway and prolactin signaling 
pathway, which indicated that the two herbs were capable of treating viral infections and the clinical manifestations relevant to various abdominal cancers, such as jaundice, abdominal pain and carbuncle[75]. It is well recognized that if jaundice is caused by pancreatic or biliary tract cancers, the most common symptom of the disease is abdominal pain[76]. Hematochezia refers to free blood in the stool, the cause of which mainly lies in the lower gastrointestinal (GI) tract, and is usually accompanied by severe, cramping lower abdominal pain[77]. A carbuncle is a red, swollen, and painful cluster of boils that are connected to each other under the skin. In fact, jaundice, hematochezia and carbuncle were 3 symptoms always accompanied with each other in poison-related conditions, such as animal poison induced by neurotoxins, viral and bacteria[78], food poison caused by fungi[79], and cancer with metastasis[80, 81], which were closely associated with human immune system.

\subsection{Molecular mechanisms of novel pain subtypes revealed by herb pharmacological actions}

As a particular personalized medicine for chronic disease management, clinical herb treatment would usually be prescribed according to the stratification of patients in terms of combined therapies (i.e. prescription usually with 10-20 distinct herbs). Therefore, establishing the connection between pain and herb categories would deliver novel biological insights for the understanding of molecular mechanisms for pain subtypes. In fact, our work on pain related herb pharmacology presented great consistency with those of recent work on the novel pharmacological and pathological mechanisms of 
pain disorders. For example, the relevant pain disease modules (Table 3), such as M94 $(p$-value $=5.15 \mathrm{E}-17)$ and M143 $(p$-value=8.61E-05), contained the recent novel pain targets P2RX7 and NK1R[82] respectively, which were also the enriched modules for herb targets. It has reported that the signaling of NK1R induced sustained excitation of spinal neurons and pain transmission, thus providing effective and sustained pain relief[82], which agreed with the efficacy of herb chili peppers (it enriched in the neuroactive ligand-receptor interaction pathway including NK1R, $p$-value $=0.01$ ). In addition, the M64 pain disease module ( $p$-value $=0.0234)$ containing one target (i.e. ATF1) of MIR-183 cluster, which controls both basal mechanical and neuropathic pain[83]. This indicated that our detected herb categories would propose a novel drugscreening source for personalized pain treatment.

Furthermore, our results delivered novel understanding of the underlying mechanisms of pain subtypes. For example, Broadleaf Holly Leaf (Ku Dingcha), which was a kind of tea used in daily life in China and was a common TCM used for detoxification, bactericidal anti-inflammation, and antioxidation effects and lipid reduction[84], was identified as a pain relief medicine in HC3. Its targets GABRA2, ADORA3, DRD2, ADORA2A, CHRNA4, FSHB were enriched in neuroactive ligandreceptor interaction pathway ( $p$-value $=0.013$ ), which were very similar to those of caffeine for sleep modulations. In recent work, caffeine has been reported to increase alertness by upregulation of the expression of DRD2 and increase of its binding, thus significantly blocking the sleep-deprivation induced mechanical pain 
hypersensitivity[85]. Further, the dopamine receptor DRD2 was found to functionally interact with the adenosine receptor ADORA2A to affect anxiogenic responses to caffeine[86], and to interact to the nicotinic acetylcholine receptor CHRNA4 to impact cognitive function[87]. This implies the effect of B. Holly Leaf on dopaminergic transmission was related to pain relief through the shared underlying mechanisms of sleep. It is interesting that we found the well-established neuropathic pain target TRPV1 covered by several regular pain herbs from HC2, such as Cinnanmomi Cortex (Rou Gui), Cinnamomi Ramulus (Gui Zhi), and Capsici Fructus (La Jiao). Furthermore, we obtained the pain disease module M246 including TRPV1 and found that there are many cough-treating herbs targeting M246 derived from HC1. These herbs included E. Snica Stapf, Fritillary, Lepidii Semen Descurainiae Semen (Ting Lizi), Belamcandae Rhizome (She Gan), and Phragmitis Rhizoma (Lu Gen), etc. In fact, in Chinese clinical prescriptions for chronic cough treatment, the aforementioned two types of herbs were regularly combined for personalized therapies. These results were consistent with the shared pharmacological and pathogenesis mechanisms (e.g. TRPV1) between refractory chronic cough and neuropathic pain[88].

Therefore, our proposed method was expected to identify herb related pain phenotypes, which was significantly useful for precision medicine. However, screening the reliable herb molecules and finally developing novel drugs for personalized therapies for pain subtypings is a non-trivial task. The quantity and quality of related data, e.g., the drug molecules with 3D structures, the related targets by docking analysis, bacteria drug 
targets and the incomplete PPI data, still constrains our study. In the future, we will collect a more complete data set of drug molecules with 3D structures to help us conduct a more comprehensive docking analysis and obtain more related targets. Meanwhile, we will integrate more reliable bacteria databases to collect more bacteria drug targets for further analysis. Nevertheless, to validate the biological findings, we are currently working with collaborators on biological assays. We believe that our method is able to increase research productivity toward herbal drug discovery. From the successful practical use of TCM prescriptions for pain treatment, we could image that the combination therapies incorporate multiple ingredients for pain management[89] would be a promising approach that should be further investigated.

\section{Materials and methods}

\subsection{Curation of herb information and the related data sources}

Pharmacopoeia of the People's Republic of China (PPRC, 2015 edition) is a legally binding collection of standards for herbal medicines in China. 'Symptom', 'syndrome', 'efficacy' and 'disease' terms were firstly extracted from the 'drug efficacy for the disease treatment' sections of 2,040 herbal medicines in PPRC, and were manually segmented into 1,717 'symptom' terms, 423 'disease' terms and 327 'efficacy' terms by 8 TCM researchers, respectively. The Chinese 'symptom', 'disease' and 'efficacy' terms were manually translated into terms with English version by the researchers, and the 'symptom' and 'disease' terms were automatically matched against the 'symptom' 
and 'disease' terms in the MeSH[90] database and the side effect-related terms in the SIDER[91] database. It is worth to note that the non-matched 'symptom' and 'disease' terms were manually re-estimated by 10 TCM researchers, and re-matched according to the criteria, i.e., the non-matched terms were manually re-matched to the terms with narrower or equal meaning. Finally, $743 \mathrm{MeSH} / \mathrm{SIDER}$ based 'symptom' terms from MeSH/SIDER and $222 \mathrm{MeSH}$ based disease' terms were collected.

To construct a list of herbal molecules, 499 herbs with related 29,384 ingredients were retrieved from the public TCMID[92], TCM Database @Taiwan[93] and HIT[94] database. The collected ingredients were matched against the compounds in the NCBI PubChem Compound Database [95] to finally obtain 1978 normalized herbal molecules. To confirm the active molecules investigated by published literatures, we integrated the SemRep database, which is a large-scale high-quality semantic knowledge resource incorporating medical concepts (e.g. phenotypes, molecules and genes) and their semantic relations derived from PubMed database using natural language processing techniques[25].

To further help measure the interactions between the pharmacological mechanism of herbs and the underlying molecular mechanisms of pain disorders, we integrated two well-established genotype-phenotype associations, namely Pain Genes Database[93] (434 genes) and Human Pain Genes Databases (172 genes, https://diatchenko.lab.mcgill.ca/lab/) on pain disorders. Finally, we collected 554 painrelated genes (termed Pgenes) for further analysis. In addition, 267 pain-related targets 
(termed Ptargets) and 21 opioid drug targets (termed Otargets) were screened from 1636 small-molecule targets in DrugBank to evaluate the interactions between pain herb and drug targets.

\subsection{Phenotype-based in silico screening of herbal active molecules}

To obtain the active herbal molecules, which have their treated phenotypes in published literatures, we integrated SemRep database to screen the co-occurrences between phenotypes and herbal molecules. 6,892 phenotype-molecule co-occurrences were retrieved involving 593 symptoms/diseases phenotypes and 406 herbal molecules. To test the reliability of the relationships, we randomly selected and manually validated 500 co-occurrences. The evaluation focused on the two key aspects: (1) whether the association between phenotypes and herbal molecule was direct and not mediated by other factors or simply coincidental co-occurrence; (2) whether the association was positive, i.e. it did not contain a negation as in "herbal molecule X was NOT related to phenotype Y". The results showed $85.4 \%$ co-occurrences were correct and meaningful, and the remaining relationships mainly included: (1) misidentification, i.e., infrequent or complicated names of molecule X; and (2) negations, i.e., molecule X cannot treat or prevent phenotype Y. This validated the reliability of the retrieved phenotype-herbal molecule relationships by literature mining.

\subsection{In silico screening of targets for herbal molecules}

We obtained the 3D structures of active herbal molecules from the PubChem database. 
Also, the 3D structures of human proteins from 936 human targets in DrugBank[26] were obtained from PDB database[96]. The small molecules for the 3D structures of human proteins were filtered by the three criteria: (1) the $3 \mathrm{D}$ structures without mutation and missing residues around the active site; (2) the 3D structures with high resolution; (3) for the selected protein structures with 2 or more chains, the single chains around the active sites were reserved for docking.

As one of the superior tools available for accurate docking, surflex flexible molecular docking method combines Hammerhead's empirical scoring function with a molecular similarity method to generate putative poses of ligand fragments. The interactions of human proteins and active herbal molecules were evaluated by Surflex-Dock search algorithm[97, 98], which were further ranked by global scores and total scores. The top $15 \%$ of the most binding proteins were finally selected as the human targets of herb molecules. In addition, owing to the strict filtering conditions to ensure the reliability of the results, the docking analysis between compounds and proteins helps us obtain only 70 related targets. However, the analysis results based on these targets are still highly credible.

16S sequence information was derived from the Human Microbiome Project (HMP), which was an international collaborative project with the aim of collecting and integrating the genomic information from multiple diverse human microbiomes. 100 stool samples were randomly selected from 1678 'normal' volunteers whose guts were inhabited by an enormous number of 'normal' microorganisms. To take into account 
the common species encountered in the healthy individuals and the capability of docking calculation, $16 \mathrm{~S}$ sequences in the 100 stool samples were first aligned with each other, and the top 450 sequences with the maximal alignment matches and the length of 100-300bp were reserved. $16 \mathrm{~S}$ rRNA is the main type of bacterial sequence information that can be obtained in public databases (i.e., HMP) at present. Therefore, in order to ensure the repeatability of prediction process in parallel and the reliability of the prediction results, we can only consider $16 \mathrm{~S}$ rRNA to represent bacteria drug targets. Furthermore, the selected $16 \mathrm{~S}$ sequences were transformed into the secondary structures of $16 \mathrm{~S}$ rRNA by RNAstructure 5.8 based on different types of experiment mapping data, and then the tertiary structures of $16 \mathrm{~S}$ rRNA by RNAComposer[99]. All the 16S rRNA-herbal molecule interaction candidates were ranked by global scores and total scores, and the top $15 \%$ of the most binding RNA were retained.

As one of the most heavily used sequence analysis tools available in the public domain, Basic Local Alignment Search Tool (BLAST) was employed to search the bacteria species of the most binding RNA, and the corresponding host targets of those bacteria species were identified from the research literature and from PATRIC database, the bacterial bioinformatics resource with a focus on human pathogenic species.

\subsection{Reliability validation of herb targets}

To obtain more reliable associations between herbs and docked targets, we screened docked herb-target associations. After docked, we obtain 21726 herb-target associations, which contain 474 herbs and 80 targets. Because too little targets (80 
targets) of docking, we extended one-order neighbors of each target. By integrating the associations between herbs and phenotypes (symptoms and diseases) and between phenotypes and genes, and adopting chi-square test to calculate the p-values of these associations, reliable associations ( $\mathrm{p}$-value $<0.05)$ between herbs and genes can be identified. Then by overlapping both of herb-target associations, 1802 reliable herbtarget associations including 238 herbs and 343 targets were retained.

The tacit assumption of herb-related mechanism is that herb pairs with shared symptoms or efficacies imply shared targets. In order to validate this assumption in our data, we firstly calculated the symptom-based or efficacy-based cosine similarity of herb pairs (238 distinct herb with targets) and the target-based cosine similarity of herb pairs. After that we obtained the correlation between these two kinds of measurements compared with the random expectations.

For example, for the given herb $i$, it can be represented by the symptom vector $V_{i}=$ $\left(w_{i, 1}, \ldots, w_{i, j}, \ldots, w_{i, n}\right)$, where $w_{i, j}=1$ if the symptom $j$ belong to the herb $i$, if not, $w_{i, j}=0$. Then the symptom-based similarity of the herb pair $x$ and $y$ can be measured by the cosine value of their symptom vectors, as formula:

$$
\operatorname{Cos}\left(V_{x}, V_{y}\right)=\frac{V_{x} \cdot V_{y}}{\left|V_{x}\right| \cdot\left|V_{y}\right|}
$$

Similarly, efficacy-based and target-based herb similarity also can be measured. To illustrate overlap results of herb pairs, we compared the overlap results with random expectation. And we reshuffled (random permutation) the targets of each herb using the Fisher-Yates method[100]. In detail, for herb pairs, remaining constant known 
efficacies and symptoms of herbs, we shuffled randomly equal numbers of targets for each herb. Then, based on the reshuffled targets of herb pairs, the overlap results can be calculated. Finally, the above procedures were repeated for 100 times, and the average ratios of overlap results can be obtained.

\subsection{Herb clustering analysis using phenotype features}

To identify the distinct herb categories with similar treated symptoms or efficacies, we constructed an herb network and applied a community detection method to detect the herb categories with distinct pain phenotypes. Based on symptoms and efficacies of herbs extracted from PPRC, out of 474 pain-related herbs, 426 herbs with 264 distinct symptoms and 573 distinct efficacies were identified. Then we calculated symptom and efficacy-based cosine similarity of herb pairs. Given the herb $i$, it can be represented by the symptom and efficacy vector $V_{i}=\left(w_{i, 1}, \ldots, w_{i, j}, \ldots, w_{i, n}\right)$, where $w_{i, j}=1$ if the symptom or efficacy $j$ belong to the herb $i$, if not, $w_{i, j}=0$. Then similarity of herb pairs can be measured by the cosine value of their vectors (Eq. 1). Finally, we obtained an herb network including 426 herbs as nodes and 25126 herb pairs with positive cosine similarity as links. To detect the distinct herb categories, here, we employed the fast unfolding algorithm[101] with wide applications to detect community structure of the herb network. Finally, the herb network was partitioned into three clusters (i.e. herb categories, modularity 0.18) containing 146 herbs, 165 herbs and 115 herbs, respectively. 


\subsection{Disease module detection of pain subtypes}

Disease module has been recognized as one of the main approaches to elucidate the underlying mechanisms of complex disease phenotypes[102]. Detecting the topological modules (or community structures) from the PPI network that are comprised of nodes that densely connect its members and are sparsely connected with the nodes in other modules[103, 104] is a basic step. We used a widely community detection algorithm called BGLL[105] based on modularity evaluation, to detect the topological modules of the PPI network. We used the STRING V9 database [106] to collect a high-quality human PPI network using interaction score threshold $(>=700$, which is suggested by the data owner), which resulted in 15,524 proteins and 218,409 links (protein-protein interaction). Although BGLL is a rather fast algorithm, it would generate the large modules with the high number (e.g. 1000) of nodes, which would induce bias for module-based analysis results. Therefore, we iteratively ran BGLL to divide the modules with over 500 nodes, which obtained the modules with no larger than 500 nodes. Meanwhile, the modules with number of nodes less than 5 were neglected. Finally, the PPI network was partitioned into 314 topological modules $(13,733$ distinct proteins), and each module contains 5 to 305 proteins. Finally, we conducted the fisher exact test to identify the enriched significant topological modules $(p$-value $<0.05)$ that are relevant to pain drugs/herb categories and pain phenotypes.

\subsection{Shortest path analysis}


To investigate the associations between different target sets (e.g. herb-related targets and pain-related targets), average shortest path length (ASPL) and shortest path length (SPL) distribution were conducted. First, for two target sets $A$ and $B$, all the shortest path lengths $\operatorname{SPL}(a, b),(a \in A, b \in \mathrm{B})$ are calculated in the protein-protein interaction (PPI) network. Then the ASPL can be calculated according to the formula:

$$
\operatorname{ASPL}(A, B)=\frac{1}{|A| \cdot|B|} \sum_{a \in A, b \in B} \operatorname{SPL}(a, b)
$$

where $|A|$ and $|B|$ represented the target number of the target set $A$ and $B$ in the PPI network, respectively.

To detect the observed characteristics of the interactions of herb targets and pain targets, we compared the distribution of them to the background distribution of all the protein pairs in PPI network. Furthermore, to clarify the significance of the distribution, these distributions are also compared with permutations. We randomly selected the $m$ (target number of $A$ ) and $\mathrm{n}$ (target number of $B$ ) targets from the PPI network, respectively and calculated the SPLs for each pair in these permutation samples (1000 times). Finally, the average distribution of random expectation is obtained from the permutation samples.

\subsection{Statistical analysis to evaluate the enriched characteristics of herb categories}

We used the statistical software R 3.1.0 to calculate most of the analytical results relevant to correlations and enriched characteristics. For example, we used chi-squared test to obtain significant herb-genes associations, and applied binomial test for 
identifying enriched phenotypes and targets of herb categories. Here, we introduce two enriched analysis methods in detail as follows.

Pathway/GO enrichment analysis. To investigate the functional characteristics of pain targets, we performed pathway and GO enrichment analysis on pain targets. The DAVID[107, 108] provides a comprehensive set of functional annotation tools for investigators to understand biological meaning behind large list of genes. We obtained the specifically associated (or enriched) pathways and GO terms with a threshold of corrected p-value $<0.05$.

Phenotype/target enrichment analysis. To identify the significant phenotypic features (i.e. indications and efficacies) and typical targets involved in each herb category, and risk ratio (RR) analysis and binomial test are used to identify the significant phenotypes and typical targets. We take identifying typical targets as an example to illuminate enrichment process. Given a target t (e.g. PRKCD) and an herb category HC (e.g. HC1), we suppose the $\mathrm{HC}$ contains $m$ herbs, and there are $n$ herbs in all the HCs. If the target $t$ is targeted by $k$ herbs in HC, and it is targeted by $s$ herbs in all the HCs. The RR of the target $t$ in $\mathrm{HC}$ can be given:

$$
\mathrm{RR}(\mathrm{t} ; \mathrm{HC})=\frac{k \cdot n}{m \cdot s}
$$

The larger RR is, more typical the target is for the HC. In addition, the p-value of $t$ in the $\mathrm{HC}$ can be calculated by binomial test. By limiting p-value $(<0.05)$ and $\mathrm{RR}(>$ 1.0), the typical targets of each herb category can be identified. Similarly, The RR analysis and binomial test were also adopted to detect the significant indications and 
efficacies for each herb category.

\section{Author contributions:}

H.S., J.C. and X.Z. conceived and designed the experiments; X.X., K. Y. and F. Z. performed the experiments; X.X., K. Y., F. Z., W. L, C.Y. Y., J.Y. W. and K.Z. collected and analyzed the data; X.Z., D. T., C. Z.,E. W. and G. R. revised the manuscript.

\section{Competing interests}

The authors declare no competing financial interests.

\section{Acknowledgements:}

This work was supported by the National Science Foundation of China (61105055, 81230086), the second batch of national "Ten thousand talents plan" of scientific and technological innovation leading talents (W02020052), and the National Key Research and Development Program (2017YFC1703506).

\section{References}

[1] J.L. Dieleman, R. Baral, M. Birger, et al., US Spending on Personal Health Care and Public Health, 1996-2013, Jama 316(24) (2016) 2627-2646.

[2] A. Manglik, H. Lin, D.K. Aryal, et al., Structure-based discovery of opioid analgesics with reduced side effects, Nature 537(7619) (2016) 185-190.

[3] R.K. Portenoy, Treatment of Cancer Pain, Drug Evaluation 377(9784) (2011) 2236-2247.

[4] D.C. Turk, H.D. Wilson, A. Cahana, Treatment of chronic non-cancer pain, Lancet 377(9784) (2011) 2226-2235.

[5] K.P. Hill, Medical Marijuana for Treatment of Chronic Pain and Other Medical and Psychiatric Problems: A Clinical Review, Journal of the American Medical Association 313(24) (2015) 2474-2483. 
[6] P. Stéphane Mélik, R. Cyril, R. William, et al., Opioid and chemokine receptor crosstalk: a promising target for pain therapy?, Nature Reviews Neuroscience 16(2) (2015) 69-78.

[7] M. Costigan, C.J. Woolf, Pain: Molecular mechanisms, Journal of Pain Official Journal of the American Pain Society 1(3) (2000) 35-44.

[8] R.R. Ji, T. Kohno, K.A. Moore, et al., Central sensitization and LTP: do pain and memory share similar mechanisms?, Trends in Neurosciences 26(12) (2003) 696705.

[9] G. Sébastien, M. Aashish, A.C. Kruse, et al., Structure of the $\delta$-opioid receptor bound to naltrindole, Nature 485(7398) (2012) 400-404.

[10] D.J. Salzberg, M.R. Weir, COX-2 Inhibitors And Cardiovascular Risk, Subcellular biochemistry 42 (2007) 159-174.

[11] J.V. Holbech, F.W. Bach, N.B. Finnerup, et al., Pain phenotype as a predictor for drug response in painful polyneuropathy_ A retrospective analysis of data from controlled clinical trials, Pain 157(6) (2016) 1305-1313.

[12] J.C. Li, X.F. Shen, X.L. Meng, et al., Analgesic effect and mechanism of the three TCM-herbal drug-combination Tou Feng Yu pill on treatment of migraine, Phytomedicine 18(8-9) (2011) 788-94.

[13] R.K. Cady, J. Goldstein, R. Nett, et al., A double-blind placebo-controlled pilot study of sublingual feverfew and ginger (LipiGesic $M$ ) in the treatment of migraine, Headache 51(7) (2011) 1078-86.

[14] L.H. Leung, E. Clementi, W.S.F. Wong, Advanced technologies charting a new path for traditional Chinese medicine drug discovery, Pharmacological Research 117 (2017) 65-66.

[15] Y. Tu, The discovery of artemisinin (qinghaosu) and gifts from Chinese medicine, Nature Medicine 17(10) (2011) 1217-20.

[16] S.L. Ingram, Pain: novel analgesics from traditional Chinese medicines, Current Biology 24(3) (2014) R114-6.

[17] J.W. Lee, W.B. Lee, W. Kim, et al., Traditional herbal medicine for cancer pain: a systematic review and meta-analysis, Complement Ther Med 23(2) (2015) 26574.

[18] X. Liang, H. Li, S. Li, A novel network pharmacology approach to analyse traditional herbal formulae: the Liu-Wei-Di-Huang pill as a case study, Molecular BioSystems 10(5) (2014) 1014-1022.

[19] J. Zheng, M. Wu, H. Wang, et al., Network pharmacology to unveil the biological basis of health-strengthening herbal medicine in cancer treatment, Cancers 10(11) (2018) 461.

[20] J. Gao, K. Zhang, Y. Wang, et al., A machine learning-driven study indicates emodin improves cardiac hypertrophy by modulation of mitochondrial SIRT3 signaling, Pharmacological Research 155 (2020) 104739.

[21] X. Zhou, J. Menche, A.L. Barabasi, et al., Human symptoms-disease network, Nat Commun 5 (2014) 4212. 
[22] A.L. Hopkins, Network pharmacology, Nature biotechnology 25(10) (2007) 1110-1111.

[23] A.L. Hopkins, Network pharmacology: the next paradigm in drug discovery, Nature chemical biology 4(11) (2008) 682-690.

[24] E. Guney, J. Menche, M. Vidal, et al., Network-based in silico drug efficacy screening, Nat Commun 7 (2016) 10331.

[25] T.C. Rindflesch, M. Fiszman, The interaction of domain knowledge and linguistic structure in natural language processing: interpreting hypernymic propositions in biomedical text, J Biomed Inform 36(6) (2003) 462-77.

[26] D.S. Wishart, C. Knox, A.C. Guo, et al., DrugBank: a comprehensive resource for in silico drug discovery and exploration, Nucleic acids research 34(suppl_1) (2006) D668-D672.

[27] B.A. Maguire, Inhibition of bacterial ribosome assembly: a suitable drug target?, Microbiology and molecular biology reviews : MMBR 73(1) (2009) 22-35.

[28] D.N. Wilson, Ribosome-targeting antibiotics and mechanisms of bacterial resistance, Nature Reviews Microbiology 12(1) (2014) 35-48.

[29] M. Campillos, M. Kuhn, A.C. Gavin, et al., Drug target identification using sideeffect similarity, Science 321(5886) (2008) 263-6.

[30] N. Rappaport, N. Nativ, G. Stelzer, et al., MalaCards: an integrated compendium for diseases and their annotation, Database 2013 (2013).

[31] M.H. van den Beuken-van Everdingen, A. de Graeff, J.L. Jongen, et al., Pharmacological Treatment of Pain in Cancer Patients: The Role of Adjuvant Analgesics, a Systematic Review, Pain Pract 17(3) (2017) 409-419.

[32] B.R. da Costa, S. Reichenbach, N. Keller, et al., Effectiveness of non-steroidal anti-inflammatory drugs for the treatment of pain in knee and hip osteoarthritis: a network meta-analysis, Lancet 387(10033) (2016) 2093-105.

[33] D. Gao, L.Y. Wu, Y.H. Jiao, et al., The effect of Xuefu Zhuyu Decoction on in vitro endothelial progenitor cell tube formation, Chin J Integr Med 16(1) (2010) 50-3.

[34] Y. Chen, G. Ke, D. Han, et al., MicroRNA-181a enhances the chemoresistance of human cervical squamous cell carcinoma to cisplatin by targeting PRKCD, Experimental cell research 320(1) (2014) 12-20.

[35] N. Hayez, I. Harfi, R. Lema-Kisoka, et al., The neuropeptides vasoactive intestinal peptide (VIP) and pituitary adenylate cyclase activating polypeptide (PACAP) modulate several biochemical pathways in human leukemic myeloid cells, Journal of neuroimmunology 149(1-2) (2004) 167-181.

[36] G. Vazquez-Ortiz, P. Pina-Sanchez, K. Vazquez, et al., Overexpression of cathepsin F, matrix metalloproteinases 11 and 12 in cervical cancer, BMC cancer 5(1) (2005) 68.

[37] J.T. Huse, E.C. Holland, Targeting brain cancer: advances in the molecular pathology of malignant glioma and medulloblastoma, Nature reviews cancer 10(5) (2010) 319-331. 
[38] M. Zöller, CD44: can a cancer-initiating cell profit from an abundantly expressed molecule?, Nature reviews cancer 11(4) (2011) 254-267.

[39] I. Avis, A. Martínez, J. Tauler, et al., Inhibitors of the arachidonic acid pathway and peroxisome proliferator-activated receptor ligands have superadditive effects on lung cancer growth inhibition, Cancer Research 65(10) (2005) 4181-4190.

[40] C. Calabrese, M. Triggiani, G. Marone, et al., Arachidonic acid metabolism in inflammatory cells of patients with bronchial asthma, Allergy 55 (2000) 27-30.

[41] I.M. Chiu, B.A. Heesters, N. Ghasemlou, et al., Bacteria activate sensory neurons that modulate pain and inflammation, Nature 501(7465) (2013) 52-7.

[42] G. Irusta, D. Abramovich, F. Parborell, et al., Direct survival role of vascular endothelial growth factor (VEGF) on rat ovarian follicular cells, Mol Cell Endocrinol 325(1-2) (2010) 93-100.

[43] M.L. Cho, Y.O. Jung, Y.M. Moon, et al., Interleukin-18 induces the production of vascular endothelial growth factor (VEGF) in rheumatoid arthritis synovial fibroblasts via AP-1-dependent pathways, Immunol Lett 103(2) (2006) 159-66.

[44] M. Zhou, M.M. McFarland-Mancini, H.M. Funk, et al., Toll-like receptor expression in normal ovary and ovarian tumors, Cancer Immunol, Immunother 58(9) (2009) 1375-85.

[45] T. Liu, Y.J. Gao, R.R. Ji, Emerging role of Toll-like receptors in the control of pain and itch, Neurosci Bull 28(2) (2012) 131-44.

[46] X.J. Liu, T. Liu, G. Chen, et al., TLR signaling adaptor protein MyD88 in primary sensory neurons contributes to persistent inflammatory and neuropathic pain and neuroinflammation, Scientific Reports 6 (2016) 28188.

[47] J. Parkash, K. Asotra, Calcium wave signaling in cancer cells, Life Sci 87(19-22) (2010) 587-595.

[48] P. Limonta, M. Montagnani Marelli, S. Mai, et al., GnRH receptors in cancer: from cell biology to novel targeted therapeutic strategies, Endocr Rev 33(5) (2012) 784-811.

[49] M.A. Olayioye, R.M. Neve, H.A. Lane, et al., The ErbB signaling network: receptor heterodimerization in development and cancer, EMBO J 19(13) (2000) 3159-67.

[50] J. Menche, A. Sharma, M. Kitsak, et al., Disease networks. Uncovering diseasedisease relationships through the incomplete interactome, Science 347(6224) (2015) 1257601.

[51] S. Begum, B. Saxena, M. Goyal, et al., Study of anti-inflammatory, analgesic and antipyretic activities of seeds of Hyoscyamus niger and isolation of a new coumarinolignan, Fitoterapia 81(3) (2010) 178-84.

[52] L. Manchikanti, S. Helm, 2nd, B. Fellows, et al., Opioid epidemic in the United States, Pain Physician 15(3 Suppl) (2012) ES9-38.

[53] H. Reinecke, C. Weber, K. Lange, et al., Analgesic efficacy of opioids in chronic pain: recent meta-analyses, Br J Pharmacol 172(2) (2015) 324-33.

[54] S. Pain, Painful progress, Nature 535(7611) (2016) S18-9. 
[55] R.A. Rudd, P. Seth, F. David, et al., Increases in Drug and Opioid-Involved Overdose Deaths - United States, 2010-2015, MMWR Morb Mortal Wkly Rep 65(5051) (2016) 1445-1452.

[56] R.S. Perez, P.E. Zollinger, P.U. Dijkstra, et al., Evidence based guidelines for complex regional pain syndrome type 1, BMC neurology 10(1) (2010) 20.

[57] R. Baron, A. Binder, G. Wasner, Neuropathic pain: diagnosis, pathophysiological mechanisms, and treatment, The Lancet Neurology 9(8) (2010) 807-819.

[58] S.M. Rubinstein, M. van Middelkoop, T. Kuijpers, et al., A systematic review on the effectiveness of complementary and alternative medicine for chronic nonspecific low-back pain, European Spine Journal 19(8) (2010) 1213-1228.

[59] G. Helft, J.-L. Georges, X. Mouranche, et al., Outcomes of primary percutaneous coronary interventions in nonagenarians with acute myocardial infarction, International journal of cardiology 192 (2015) 24-29.

[60] I.-S. Kim, Y.-J. Park, S.-J. Yoon, et al., Ephedrannin A and B from roots of Ephedra sinica inhibit lipopolysaccharide-induced inflammatory mediators by suppressing nuclear factor- $\mathrm{KB}$ activation in RAW 264.7 macrophages, International immunopharmacology 10(12) (2010) 1616-1625.

[61] C. Brightling, M. Berry, Y. Amrani, Targeting TNF- $\alpha$ : a novel therapeutic approach for asthma, Journal of Allergy Clinical Immunology 121(1) (2008) 510.

[62] P. Bhavsar, N. Khorasani, M. Hew, et al., Effect of p38 MAPK inhibition on corticosteroid suppression of cytokine release in severe asthma, European Respiratory Journal 35(4) (2010) 750-756.

[63] Z.-D. Yang, D.-Z. Duan, A new alkaloid from Fritillaria ussuriensis Maxim, Fitoterapia 83(1) (2012) 137-141.

[64] L.A. Shimoda, M. Fallon, S. Pisarcik, et al., HIF-1 regulates hypoxic induction of NHE1 expression and alkalinization of intracellular pH in pulmonary arterial myocytes, American Journal of Physiology-Lung Cellular Molecular Physiology 291(5) (2006) L941-L949.

[65] J.H. Boyd, E.A. Macklin, R.C. Strunk, et al., Asthma is associated with acute chest syndrome and pain in children with sickle cell anemia, Blood 108(9) (2006) 2923-7.

[66] W. Yin, T.-S. Wang, F.-Z. Yin, et al., Analgesic and anti-inflammatory properties of brucine and brucine N-oxide extracted from seeds of Strychnos nux-vomica, J Ethnopharmacol 88(2-3) (2003) 205-214.

[67] T. Gao, J. Hao, Z. Wiesenfeld-Hallin, et al., Analgesic effect of sinomenine in rodents after inflammation and nerve injury, European journal of pharmacology 721(1-3) (2013) 5-11.

[68] A.A. Grom, J. Villanueva, S. Lee, et al., Natural killer cell dysfunction in patients with systemic-onset juvenile rheumatoid arthritis and macrophage activation syndrome, The Journal of pediatrics 142(3) (2003) 292-296. 
[69] M. Clodi, G. Vila, R. Geyeregger, et al., Oxytocin alleviates the neuroendocrine and cytokine response to bacterial endotoxin in healthy men, American Journal of Physiology-Endocrinology Metabolism 295(3) (2008) E686-E691.

[70] Y. Kochi, R. Yamada, A. Suzuki, et al., A functional variant in FCRL3, encoding Fc receptor-like 3, is associated with rheumatoid arthritis and several autoimmunities, Nature genetics 37(5) (2005) 478-485.

[71] T.S.P.C.o.P.s.R.o. China, Pharmacopoeia of the people's republic of china, People's Medical Publishing House 2005.

[72] T. Iizuka, M. Nagai, Vasorelaxant effects of forsythiaside from the fruits of Forsythia suspensa, Yakugaku zasshi: Journal of the Pharmaceutical Society of Japan 125(2) (2005) 219-224.

[73] S.-J. Dai, Y. Ren, L. Shen, et al., New alkaloids from Forsythia suspensa and their anti-inflammatory activities, Planta medica 75(04) (2009) 375-377.

[74] W.-L. Jiang, F.-H. Fu, B.-M. Xu, et al., Cardioprotection with forsythoside B in rat myocardial ischemia-reperfusion injury: relation to inflammation response, Phytomedicine 17(8-9) (2010) 635-639.

[75] P. Zhang, M. Yang, Y. Zhang, et al., Dissecting the single-cell transcriptome network underlying gastric premalignant lesions and early gastric cancer, Cell reports 27(6) (2019) 1934-1947. e5.

[76] J.M. Regimbeau, D. Fuks, P. Bachellier, et al., Prognostic value of jaundice in patients with gallbladder cancer by the AFC-GBC-2009 study group, Eur J Surg Oncol 37(6) (2011) 505-12.

[77] J.M. Inadomi, S. Vijan, N.K. Janz, et al., Adherence to colorectal cancer screening: a randomized clinical trial of competing strategies, Arch Intern Med 172(7) (2012) 575-82.

[78] M.C. Hardy, J. Cochrane, R.E. Allavena, Venomous and Poisonous Australian Animals of Veterinary Importance: A Rich Source of Novel Therapeutics, Biomed Research International 2014 (2014) 671041.

[79] D.M. Dereszynski, S.A. Center, J.E. Randolph, et al., Clinical and clinicopathologic features of dogs that consumed foodborne hepatotoxic aflatoxins: 72 cases (2005-2006), Javma-Journal Of the American Veterinary Medical Association 232(9) (2008) 1329-1337.

[80] A. Sevinc, S. Buyukberber, C. Camci, et al., Granulocytic sarcoma of the colon and leukemic infiltration of the liver in a patient presenting with hematochezia and jaundice, Digestion 69(4) (2004) 262-5.

[81] M. Watanabe, K. Shiozawa, T. Mimura, et al., Hepatic artery pseudoaneurysm after endoscopic biliary stenting for bile duct cancer, World J Radiol 4(3) (2012) 115-20.

[82] D.D. Jensen, T. Lieu, M.L. Halls, et al., Neurokinin 1 receptor signaling in endosomes mediates sustained nociception and is a viable therapeutic target for prolonged pain relief, Sci Transl Med 9(392) (2017). 
[83] C. Peng, L. Li, M.D. Zhang, et al., MiR-183 cluster scales mechanical pain sensitivity by regulating basal and neuropathic pain genes, Science 356(6343) (2017) 1168-1171.

[84] L. Li, L.J. Xu, G.Z. Ma, et al., The large-leaved Kudingcha (Ilex latifolia Thunb and Ilex kudingcha C.J. Tseng): a traditional Chinese tea with plentiful secondary metabolites and potential biological activities, J Nat Med 67(3) (2013) 425-37.

[85] C. Alexandre, A. Latremoliere, A. Ferreira, et al., Decreased alertness due to sleep loss increases pain sensitivity in mice, Nature Medicine 23(6) (2017) 768-774.

[86] E. Childs, C. Hohoff, J. Deckert, et al., Association between ADORA2A and DRD2 polymorphisms and caffeine-induced anxiety, Neuropsychopharmacology 33(12) (2008) 2791-800.

[87] S.A. Markett, C. Montag, M. Reuter, The association between dopamine DRD2 polymorphisms and working memory capacity is modulated by a functional polymorphism on the nicotinic receptor gene CHRNA4, J Cogn Neurosci 22(9) (2010) 1944-54.

[88] A. Niimi, K.F. Chung, Evidence for neuropathic processes in chronic cough, Pulmonary Pharmacology \& Therapeutics 35 (2015) 100-104.

[89] J.K. Baillie, I. Power, Morphine, gabapentin, or their combination for neuropathic pain, New England Journal of Medicine 352(25) (2005) 2650.

[90] C.E. Lipscomb, Medical Subject Headings (MeSH), Bulletin of the Medical Library Association 88(3) (2000) 265-266.

[91] M. Kuhn, M. Campillos, I. Letunic, et al., A side effect resource to capture phenotypic effects of drugs, Molecular Systems Biology 6(1) (2010) 343.

[92] R. Xue, Z. Fang, M. Zhang, et al., TCMID: Traditional Chinese Medicine integrative database for herb molecular mechanism analysis, Nucleic acids research 41(Database issue) (2013) D1089-95.

[93] C.Y. Chen, TCM Database@Taiwan: the world's largest traditional Chinese medicine database for drug screening in silico, PLoS One 6(1) (2011) e15939.

[94] H. Ye, L. Ye, H. Kang, et al., HIT: linking herbal active ingredients to targets, Nucleic acids research 39(Database issue) (2011) D1055-9.

[95] S. Kim, P.A. Thiessen, E.E. Bolton, et al., PubChem Substance and Compound databases, Nucleic acids research 44(D1) (2016) D1202-13.

[96] H. Berman, K. Henrick, H. Nakamura, Announcing the worldwide protein data bank, Nature Structural Molecular Biology 10(12) (2003) 980.

[97] T.E. Mansley, J.K. Shepphird, L. Wang, Evaluation of docking performance: Comparison of Surflex-Dock with five docking algorithms using the Johnson \& Johnson data set, American Chemical Society, Amer Chemical Soc 1155 16TH ST, NW, Washington, DC 20036 USA, 2007.

[98] A.N. Jain, Surflex: fully automatic flexible molecular docking using a molecular similarity-based search engine, Journal of medicinal chemistry 46(4) (2003) 499511. 
[99] M. Popenda, M. Szachniuk, M. Antczak, et al., Automated 3D structure composition for large RNAs, Nucleic acids research 40(14) (2012) e112-e112.

[100] R.A. Fisher, F. Yates, Statistical tables for biological, agricultural and medical research, Oliver and Boyd Ltd, London 1943.

[101] A. Clauset, M.E. Newman, C. Moore, Finding community structure in very large networks, Physical Review E Statistical Nonlinear \& Soft Matter Physics 70(2) (2004) 066111.

[102] J. Menche, A. Sharma, M. Kitsak, et al., Uncovering disease-disease relationships through the incomplete interactome, Science 347(6224) (2015) 1257601.

[103] Y. Wang, H. Liu, Y. Lin, et al., Network-Based Approach to Identify Potential Targets and Drugs that Promote Neuroprotection and Neurorepair in Acute Ischemic Stroke, Scientific Reports 7 (2017) 40137.

[104] M.T. Dittrich, G.W. Klau, A. Rosenwald, et al., Identifying functional modules in protein-protein interaction networks: an integrated exact approach, Bioinformatics 24(13) (2008) i223-i231.

[105] V.D. Blondel, J.L. Guillaume, R. Lambiotte, et al., Fast unfolding of community hierarchies in large networks, J Stat Mech abs/0803.0476 (2008) 1008.

[106] A. Franceschini, D. Szklarczyk, S. Frankild, et al., STRING v9. 1: protein-protein interaction networks, with increased coverage and integration, Nucleic acids research 41(D1) (2012) D808-D815.

[107] W. Huang da, B.T. Sherman, R.A. Lempicki, Systematic and integrative analysis of large gene lists using DAVID bioinformatics resources, Nature Protocols 4(1) (2009) 44-57.

[108] W. Huang da, B.T. Sherman, R.A. Lempicki, Bioinformatics enrichment tools: paths toward the comprehensive functional analysis of large gene lists, Nucleic acids research 37(1) (2009) 1-13. 